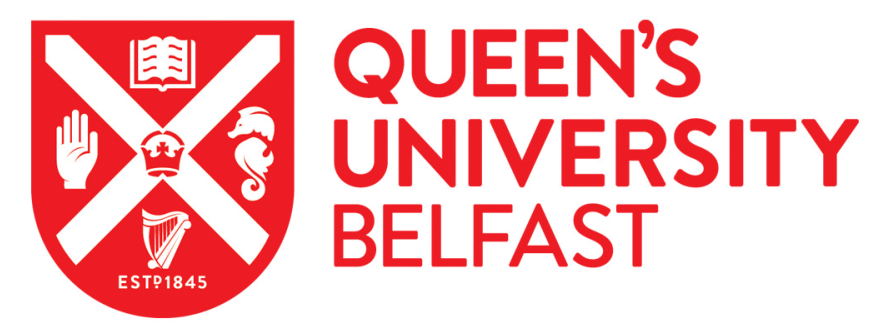

\title{
Endothelial cells derived from patients with diabetic macular edema recapitulate clinical evaluations of anti-VEGF responsiveness through the Neuronal Pentraxin 2 pathway
}

Vila-Gonzalez, M., Kelaini, S., Naderi-Meshkin, H., Margariti, A., Stewart, S., Virgili, G., Grieve, D. J., Stitt, A. W., Lois, N., \& Margariti, A. (2020). Endothelial cells derived from patients with diabetic macular edema recapitulate clinical evaluations of anti-VEGF responsiveness through the Neuronal Pentraxin 2 pathway. Diabetes. https://doi.org/10.2337/db19-1068

Published in:

Diabetes

Document Version:

Peer reviewed version

Queen's University Belfast - Research Portal:

Link to publication record in Queen's University Belfast Research Portal

Publisher rights

(c) 2020 by the American Diabetes Association.

This work is made available online in accordance with the publisher's policies. Please refer to any applicable terms of use of the publisher.

\section{General rights}

Copyright for the publications made accessible via the Queen's University Belfast Research Portal is retained by the author(s) and / or other copyright owners and it is a condition of accessing these publications that users recognise and abide by the legal requirements associated with these rights.

Take down policy

The Research Portal is Queen's institutional repository that provides access to Queen's research output. Every effort has been made to ensure that content in the Research Portal does not infringe any person's rights, or applicable UK laws. If you discover content in the

Research Portal that you believe breaches copyright or violates any law, please contact openaccess@qub.ac.uk. 
Endothelial cells derived from patients with diabetic macular edema recapitulate clinical evaluations of anti-VEGF responsiveness through the Neuronal Pentraxin 2 pathway

\author{
Marta Vila Gonzalez ${ }^{1}$ \\ Magdalini Eleftheriadou ${ }^{1}$ \\ Sophia Kelaini ${ }^{1}$ \\ Hojjat Naderi-Meshkin ${ }^{1}$ \\ Shonagh Flanagan ${ }^{1}$ \\ Stephen Stewart ${ }^{2}$ \\ Gianni Virgili \\ David J. Grieve ${ }^{1}$ \\ Alan W. Stitt ${ }^{1}$ \\ Noemi Lois ${ }^{1,2}$ \\ Andriana Margariti ${ }^{1}$
}

Wellcome-Wolfson Institute for Experimental Medicine ${ }^{1}$ and the Belfast Health and Social Care Trust ${ }^{2}$, Belfast, UK; and the University of Florence, Florence ${ }^{3}$, Italy.

Corresponding Authors: Andriana Margariti a.margariti@qub.ac.uk and Noemi Lois n.lois@qub.ac.uk; Wellcome-Wolfson Institute for Experimental Medicine, 97 Lisburn Road, Belfast, BT9 7BL, UK. 


\section{Abstract}

Diabetic macular edema (DME) remains a leading cause of vision loss worldwide. DME is commonly treated with intravitreal injections of vascular endothelial growth factor (VEGF) neutralising antibodies. Anti-VEGFs are effective but not all patients fully respond to them. Given their potential side effects, inconvenience and high cost, identifying who may not respond appropriately to anti-VEGFs and why is essential.

Herein, we determine first the response to anti-VEGFs in a cohort of DME patients using spectral-domain optical coherence tomography scans obtained throughout the first year of treatment. We found that in $28 \%$ of eyes full clearance of fluid occurred at any time during the first year ("full responders"); in 66\% fluid cleared only partly ("partial responders"); in 6\% fluid remained unchanged ("non-responders"). To understand this differential response, we generated induced pluripotent stem cells (iPS) from "full responders" and "non-responders" and from diabetic subjects with no DME and age-matched non-diabetic volunteers and differentiated them into endothelial cells (iPS-ECs). Monolayers of iPS-ECs derived from diabetics showed marked and prolonged increased permeability upon exposure to VEGF when compared with non-diabetic controls; the response was significantly exaggerated in iPSECs from "non-responders" when compared with "full responders". Moreover, phosphorylation of key cellular proteins in response to VEGF, including VEGFR2, and gene expression profiles, such as Neuronal Pentraxin 2 (NPTX2) expression, differed between "full responders" and "non-responders".

In the current study, iPS were used to predict patient response to anti-VEGF and identify key mechanisms underpinning the differential outcomes observed in the clinic. This approach has identified NPTX2 as playing a significant role in patient-linked responses and has potential as a new therapeutic target for DME. 


\section{Significance statement}

Vascular endothelial growth factor inhibitors (anti-VEGFs) are used to treat diabetic macular edema (DME). Anti-VEGFs are given as an eye injection requiring frequent and long-term treatment. The work presented herein demonstrated that in only around a quarter of eyes full resolution of DME occurs in the first year of treatment with anti-VEGFs; many do not respond adequately to this therapy. It showed also that in vitro readouts of induced pluripotent stem cell (iPS)-derived endothelial cell responses can be used to identify patient response to anti-VEGF, which has potential to improve clinical decision-making for use of alternative therapies for those unlikely to respond to anti-VEGFs. Furthermore, iPS cell technology may enable targeted drug development for DME not predominantly driven by VEGF.

Keywords: iPS, iPS-ECs, DME, endothelial cells, diabetes, diabetic retinopathy, retina, edema. 


\section{INTRODUCTION}

Diabetic macular edema (DME) remains a leading cause of visual loss in people with diabetes mellitus (DM) [1]. It results from the breakdown of the inner and outer blood retinal barriers (BRBs) with subsequent leakage of blood, fluid, lipid and infiltration of inflammatory cells in the macula, which alters retinal architecture and function [2]. Although the exact mechanisms that lead to DME are not completely understood, evidence suggests that vascular endothelial growth factor (VEGF) plays a major role, often in unison with an array of proinflammatory cytokines [3]. VEGF is elevated in the vitreous of patients with DME [4] while experimental studies have demonstrated VEGF induces vascular leakage by disrupting the integrity of endothelial cell (EC) junctional complexes and loss of barrier function [5].

Over recent years, randomised controlled trials (RCTs) have demonstrated the benefit of VEGF neutralizing antibodies to improve vision and reduce macular fluid in people with DME [6-10]. However, despite their proven efficacy, it is now evident that not all patients with DME respond to anti-VEGFs in a similar manner. To date, there is no consensus with regard to a definition of "responder" and "non-responder" to anti-VEGFs [11-14] nor is there a robust predictor for who will and will not respond to this therapy. Anti-VEGFs are given as intravitreal injections and need to be administered monthly throughout the first year of treatment in most patients, and at frequent intervals thereafter. The great majority of DME patients will require long-term treatment for the benefit of anti-VEGFs to be maintained, with $\sim 50 \%$ of patients still requiring treatment 5 years following its initiation [15]. An ability to predict patient responsiveness to this therapy would be of great benefit to avoid unnecessary risks, inconvenience and distress caused by repeated injections and would allow more timely intervention with alternative therapies [16]. Such a personalised approach to anti-VEGF administration would avoid the loss of vision that may occur as a result of selecting or continuing with an ineffective treatment, unnecessary workload to health professionals and costs to health services.

In the current study, we evaluated the response to anti-VEGFs in a large cohort of patients with DME treated with anti-VEGFs and shown that in only $28 \%$ the edema cleared fully at any time during the first year of anti-VEGF treatment. Also, having previously developed a unique approach to generate induced pluripotent stem cells (iPS) and differentiated 
endothelium from small volumes of blood [17], we designed a conjoined clinical and experimental study using iPS microvascular endothelium derived from patients with diabetic retinopathy (DR) without DME and from DME patients classified as "full responders" and "non-responders" to anti-VEGFs. Their iPS-ECs were assessed for VEGF-induced permeability responses, phosphorylation pathways and gene expression profiles. There were clear differences found between "full responders" and "non-responders" which may reflect distinct pathophysiology in ECs from the patient groups and could offer not only useful clinical information but provide scope for new drug development.

\section{SUBJECTS, MATERIALS AND METHODS}

\section{Evaluating the treatment response to anti-VEGFs in patients with DME}

Electronic records of all consecutive patients with newly diagnosed DME examined at the Belfast Health and Social Care Trust (BHSCT) were retrospectively reviewed. This study was part of an approved audit (audit approval number 5170) for which full ethical review was waived. A patient cohort was constructed. Eligibility criteria for this cohort included: 1) having received the first dose of anti-VEGF between March $1^{\text {st }}$ and December $31^{\text {st }} 2014 ; 2$ ) having been treated exclusively with ranibizumab, as this was the first anti-VEGF approved for the treatment of DME in UK and the great majority of patients at the BHCST would have received this drug during the period of the study; 3 ) having received a minimum loading dose ( 3 monthly consecutive injections); and 4) having a minimum follow-up of 12 months with regular visits (4-6 weeks).

Spectral domain optical coherence tomography (SD-OCTs) scans obtained throughout the whole first year of treatment were reviewed and graded by an expert clinician (NL) masked to clinical findings and classified as:

"Full responders" when the macula fully dried at any point during the first year or treatment (the presence of small, isolated or sparse cysts were allowed within the definition of full responders); "Partial responders" when there was a reduction in the amount of fluid present at the macula but when fluid never fully dried during the first year of treatment; "Nonresponders" when macular fluid remained unchanged on qualitative evaluation of SD-OCTs throughout the first year of treatment and, in addition, when the change in central retinal thickness (CRT) was $\leq 15 \%$ from baseline. 
Differences among the above groups in demographics (age, gender), type of diabetes mellitus (DM) (1 vs 2), best-corrected visual acuity (BCVA), number of injections received and central retinal subfield thickness (CRST) measured using SD-OCT, were investigated. Chi-square test was used to compare proportions (sex and DM type) among anatomic response categories. Linear regression was fit using Generalised Linear Models adjusting standard errors for within-patient correlation using a 'sandwich' (Huber-White) variance estimator.

Predicting a treatment response to anti-VEGFs in patients with DME using iPS-derived ECs in vitro and elucidating the molecular mechanisms involved.

This prospective study was approved by the Office for Research Ethics Committees Northern Ireland under the title of "STRatifying trEatment for diAbetic MacuLar oedema using Induced pluripotEnt stem (iPS) cells" (STREAMLINE) (REC reference 14/NI/1109; IRAS project ID 162254). Patients with diabetic retinopathy were recruited from ophthalmic clinics at the Belfast Health and Social Care Trust (BHSCT). Non-diabetic volunteers were recruited as controls from individuals accompanying patients to the clinic to the BHSCT and from Queen's University Belfast.

\section{Inclusion criteria}

Patients with diabetes and mild non-proliferative diabetic retinopathy (NPDR) with no DME $(n=6)$, those with DME classified as "full responders" $(n=6)$ and as "non-responders" $(n=6)$ to anti-VEGF therapy (Ranibizumab), and age-matched non-diabetic volunteers $(n=6)$ were recruited into the study. Verbal and written information about the study was provided to all potentially eligible participants. Informed consent was obtained prior to study procedures from those willing and consenting to take part. The study adhered to the principles of ethical medical research as detailed in the Declaration of Helsinki.

\section{Exclusion criteria}

Patients unable to provide informed consent for the study were excluded.

On the day of blood sample collection, a detailed medical and eye history was obtained and blood pressure was measured. 
A 20-ml sample of peripheral blood was obtained from all participants by venepuncture into VACUETTE ${ }^{\circledR}$ K3 EDTA-coated $4 \mathrm{ml}$ tubes (454021, GREINER Bio-one). Samples were processed in the laboratory by scientists masked to clinical findings and origin of the samples [i.e. scientists were not informed from which donor group samples were coming from].

\section{MNCs isolation and culture}

MNC isolation was performed as previously described [17]. Briefly, blood was separated by gradient by layering it on Histopaque solution (10771, SIGMA) (1:1 ratio) and centrifugation for 30 minutes at $550 \mathrm{gx}$ with the break off and at room temperature. The MNCs buffy coat was collected with a soft plastic pipette and placed in a $15 \mathrm{ml}$ tube, topped up with PBS and centrifuged for 10 minutes at $300 \mathrm{gx}$ at $4^{\circ} \mathrm{C}$. After 3 washes with PBS, the cells were resuspended in $1 \mathrm{ml}$ of MNC medium as previously described [17] and plated in MNC medium at a density of 4 million cells $/ \mathrm{ml}$. The cells were expanded for 7 days and then used for reprogramming.

\section{Generation of patient-specific iPS cells}

iPS cells were generated from patients' blood MNCs as previously described [17]. Up to 2 million MNCs were transfected with $5 \mu \mathrm{g}$ of plasmid per million cells (5:1 ratio pEB-C5:pEB$\mathrm{Tg}$ ) using the Lonza CD34 nucleofector kit (VPA-1003, Lonza) and Amaxa nucleofector (program T-016) according to the manufacturer's protocol. pEB-C5 and pEB-Tg plasmids were a gift from Linzhao Cheng (Addgene plasmids \#28213 and \#28220). On day 2 after transfection, the cells were seeded onto inactivated feeders (mouse embryonic fibroblasts from ATCC) in reprogramming medium (KnockOut ${ }^{\mathrm{TM}}$ DMEM/F-12 with MEM Non-Essential Amino Acids Solution and GlutaMAX ${ }^{\mathrm{TM}}$ supplemented with KO Serum replacement (20\%), 2Mercaptoethanol $(0.1 \mathrm{mM})$ and human recombinant fibroblast growth factor FGF2 (10 $\mathrm{ng} / \mathrm{ml}$ ), all from Thermo Fisher Scientific except for FGF2, from MACS Miltenyi Biotec). Reprogramming medium was changed every day until day. Colonies appeared from day 9. For the culture of iPS cell lines, reprogramming medium was changed every day and the iPS colonies were subcultured once per week with a 1:6 ratio.

\section{Differentiation of iPS into iPS-ECs}


iPS cells were cultured under feeder-free conditions and seeded on GFR Basement Membrane Matrix (\#354230 Corning) in StemMACS ${ }^{\mathrm{TM}}$ iPS-Brew XF (130-104-368, MACS Milteniy Biotec). Next day, the medium was replaced by Neurobasal medium (21103049, Thermo Fisher Scientific) with N-2 Supplement (17502001, Thermo Fisher Scientific) and B-27 ${ }^{\mathrm{TM}}$ Supplement (17504044, Thermo Fisher Scientific) supplemented with 25 ng/ml BMP4 (PHC9531, Thermo Fisher Scientific) and 8 MM CHIR99021 (SML1046, SIGMA). 72 later, the medium was replaced with StemPro ${ }^{\text {TM }}-34$ SFM (10639011, Thermo Fisher Scientific) supplemented with $200 \mathrm{ng} / \mathrm{ml}$ VEGF (PHC9391, Thermo Fisher Scientific) and $2 \mu \mathrm{M}$ forskolin (F6886, SIGMA). This step was repeated after 24 hours.

On day 6 of differentiation, magnetic activated cell sorting (MACS) of cells expressing the mature EC marker CD144 was performed using anti-human CD144 MicroBeads (130-097-857, MACS Miltenyi Biotec). The cells were seeded on mouse collagen IV-coated plates in EGM-2 $10 \%$

FBS media supplemented with $50 \mathrm{ng} / \mathrm{ml}$ VEGF and $10 \mu \mathrm{M}$ LY364947. Medium was replaced every other day and the cells were used for further analysis or cryopreserved once confluent.

\section{RNA extraction, RT-PCR and qRT-PCR}

Determination of relative mRNA expression of specific cell markers (and other genes of interest) was performed using RNA extraction, RT-PCR and qRT-PCR as previously described [18]. Primers for CD235, CD71, OCT4, LIN28, NANOG, CD144, CD31, VEGFR2 and NPTX2 are listed in Supplementary Table 2. GAPDH, ATP5B, ACTB and UBC were also used as reference genes (all from human GeNorm Kit ge-SY-12, Primer Design; sequences not provided by manufacturer).

\section{Western blotting}

Protein detection of specific cell markers was performed using western blot as previously described [18]. To ensure valid comparison between samples, cells were harvested at same timepoints and/or confluence (70-80\% for iPS cells, $80-100 \%$ for iPS-ECs). For the detection of phosphorylated proteins, TBS-T was used instead of PBS-T and 5\% BSA TBS-T was used as blocking solution instead of 5\% milk in PBS-T. Antibodies against TRA-1-60 (ab16288), OCT4 (ab19857), LIN28 (ab46020), NPTX2 (ab69858) from abcam, CD144 (STJ96234, St John's Laboratories), Phospho-VEGFR2 (PA5-12598) and Phospho-eNOS (PA5-35879), both from 
Thermo Fisher Scientific, were used. Antibody against actin beta (MAB8929, R\&D) or GAPDH (ab125247, abcam) was used for loading controls. Quantification of the western blots was performed using ImageJ and samples were normalised to the loading control (actin beta or GAPDH).

\section{Immunofluorescence staining}

Immunofluorescence staining procedures have been previously detailed in [17]. Live staining of iPS cells using CDy1 dye (Active Motif, 14001) was performed as indicated in the manufacturer's instructions.

\section{Migration assay}

Migration capacity was studied by performing a wound healing or scratch assay. iPS-ECs were seeded in wells of a 24-well plate at a density of 50000 iPS-ECs/well. Once confluent, the monolayer was scratched from side to side along the well using a $200 \mu \mathrm{l}$ tip. Pictures of the wound were taken at $0,4,8,12$ and 24 -hour time points. The migration capacity of the cells was calculated by measuring the change from baseline, in which the area of the wound ( 0 hours after the scratch) minus the area of the wound at different time points $(4,8,12$ and 24 hours), equalled the area that the cells had covered during that time.

\section{Proliferation assay}

Cell proliferation of iPS-ECs was assessed using CyQUANT' ${ }^{\mathrm{TM}}$ NF Cell Proliferation Assay Kit (C35006, Thermo Fisher Scientific) according to the manufacturer's instructions. iPS-ECs were seeded in 96-well plate wells at a density of 5000 cells/well and were allowed to adhere for at least 4 hours. Cells were seeded in quadruplicates for each cell line and time point $(8,24$ and 48 hours). The values at different time points were normalised to the values obtained at 8 hours for each experiment.

\section{Permeability assessment}

Permeability response to VEGF in iPS-ECs from different patients/donors was measured using xCELLigence ${ }^{\circledR}$ RTCA DP (Real-Time Cell Analyzer Dual Purpose, ACEA Biosciences Inc), which measures cell impedance in real time. 20000 cells in $200 \mu$ l were seeded per well of a 16 well polyethylene terephthalate plate pre-coated with mouse CIV. Four replicates were seeded for 
each condition. The cells reached confluence overnight. 24 hours after seeding, the cells were starved for 2 hours by replacing $150 \mu$ of medium with endothelial basal medium plus HEPES at $25 \mathrm{mM}$ (15630106, Thermo Fisher Scientific). Next, $100 \mu \mathrm{l}$ of the medium were replaced with starvation medium with 200,100 or $50 \mathrm{ng} / \mathrm{ml} \mathrm{VEGF} \mathrm{(for} \mathrm{a} \mathrm{final} \mathrm{concentration} \mathrm{of} \mathrm{100,} 50$ or $25 \mathrm{ng} / \mathrm{ml}$ ). 0.1\% BSA in PBS was used as a vehicle control. Data were analysed using RTCA Data Analysis Software and Prism 5.

\section{RNA-Sequencing}

Quality control and RNA sequencing analysis was performed by the Queen's Genomics Core Technology Unit (GCTU). In Brief, the cells were briefly washed with PBS and harvested using QIAzol lysis buffer. Sample and library preparation were done as previously described [17]. Libraries were sequenced on a NextSeq (Illumina) and paired-end reads mapped to the human reference genome (hg38), allowing up to 2 mismatches and up to 10 hits per read using the CLC Genomics Workbench 10.0.1 (https://www.qiagenbioinformatics.com). Reference sequences were annotated with genes and transcripts. Reference content was mapped to gene regions only and the raw read counts were generated by HTSeq-Counts. To identify differentially expressed genes (DEGs) between responder and non-responder samples of RNA-seq, a differential expression analysis was performed using R package DESeq2, followed by standard visualization using EnhancedVolcano and Pheatmap in R. Comparative gene expression data was filtered according to the absolute log 2 fold change greater than +1 and adjusted $p$-value (q-value) threshold of $<0.05$.

\section{Lentiviral particle transduction and VEGF treatment}

Lentiviral particles were produced using MISSION shNT or shNPTX2 plasmids (REFERENCES? Sigma-Aldrich) according to the protocol previously described [18-20]. The efficiency of the infection was $70-80 \%$. Two hours before harvesting, the cells were serum-depleted and treated with $50 \mathrm{ng} / \mathrm{ml}$ of VEGF 5 minutes and 20 minutes before harvesting. Vehicle control cells (0.1\% BSA in PBS), harvested at exactly 72 hours, served as the control (0 minutes timepoint). 


\section{FITC-Dextran treatment}

iPS-ECS from "non-responders" seeded in transwell plates were treated with $1 \mathrm{mg} / \mathrm{ml}$ Fluorescein isothiocyanate-dextran 4 (\#46944 Sigma-Aldrich) between 3 and 24 hours after transduction with shNPTX2 or shNT. Fluorescence was determined at $520 \mathrm{~nm}$ emission and $485 \mathrm{~nm}$ excitation and relative percent diffusion was calculated.

\section{Statistical analysis}

Data in graphs are expressed as the mean \pm SEM and were analysed using GraphPad Prism 5 software with a two-tailed Student's t test for two groups or pairwise comparisons or ANOVA. A value of $p<0.05$ was considered significant. Values were considered significant when * $p<0.05, * * p<0.01$ or $* * * p<0.001$.

"The datasets generated and/or analyzed during the current study are available from the corresponding authors upon reasonable request".

"The patient specific iPS cell lines generated during the current study are available from the corresponding authors upon reasonable request".

\section{RESULTS}

\section{Evaluating the treatment response to anti-VEGFs in patients with DME.}

One hundred and forty eyes of 100 patients with DME treated with Ranibizumab formed the study cohort. Colour fundus photographs of the right eye were obtained from an individual with no retinal disease (Figure $1 \mathrm{~A}$, top, left) and from a patient with DME (Figure $1 \mathrm{~A}$, top, right). Hard exudation is seen involving the macula and extending to the fovea (black arrow). There were 172 eyes treated during the study period; 32 were excluded as they did not meet the eligibility criteria for the study $[<12$ month follow-up or use of other intravitreal therapy (i.e. aflibercept or steroids)].

The mean BCVA was 59.7 ETDRS letters (SD 15.4) at baseline and improved to 67.3 EDTRS letters (SD 14.4) at 12 months follow-up, after a mean number of 7.5 (SD 2.04) Ranibizumab injections. The anti-VEGF response observed and demographics and clinical characteristics of 
patients (eyes) included in the study have been summarised in Supplementary Tables 3 and 4. An example of a "full responder" and a "non-responder" is shown in Figure 1B. In "full responders" the macula dried after a median time of 7 months (range 1-12 months).

There were no differences among groups in any of the clinical parameters evaluated other than, as expected, in CRST and in the number of intravitreal anti-VEGF injections received, with "partial responders" and "non-responders" receiving a higher number of injections when compared with "full responders". In 39 patients in the cohort both eyes were eligible and, thus, included in the study. Of these, in 28 (72\%) concordance of the response between eyes was observed (i.e. right eyes were in the same category of anti-VEGF response than left eyes).

\section{Predicting a treatment response to anti-VEGFs in patients with DME using iPS-ECs in vitro.}

Demographics and characteristics of participants are detailed in Supplementary Table 1.

iPS cells generated from non-diabetic and diabetic donors showed identical reprogramming and differentiating capacities. These iPS cell lines were initially generated from 3 subjects from each cohort [patients with DR and no DME; patients with DR and DME that responded fully to anti-VEGF treatment ("full responders", from here on referred to as "responders") and those that did not respond to this therapy ("non-responders") and from age-matched healthy volunteers]. For each group, one iPS cell line was selected, expanded and characterised (Figure 2) before being differentiated into ECs (iPS-ECs) (Figures 3 and 4). Established iPS colonies were examined for pluripotency using qRT-PCR (Figure 2A-B) and immunoblotting (Figure 2C) which confirmed high expression of pluripotency markers and low expression of MNC markers at both RNA and protein level. iPS colonies also stained positive for CDy1, Oct4, Lin28, and TRA-1-60 (Figure 2D). Overall, iPS from the different groups were comparable in terms of expression of pluripotency markers as well as morphology, indicating the reprogrammable nature of each cohort's samples.

Central to this study was the comparison between patients with DME; therefore, to avoid misidentification and rule out possible cross contamination of cell lines, a Short Tandem Repeat (STR) profiling of the iPS cell lines was undertaken (Supplementary Table 5). When two cell lines shared less than $56 \%$ of the analysed alleles they could be considered to come from different individuals and the selected iPS cell lines met these criteria (Supplementary Table 5 and Supplementary Table 6) confirming that they came from different donors. 
Moreover, none of the iPS matched any of the lines recorded by The European Collection of Authenticated Cell Cultures (ECACC). This provided important assurance that the iPS cells used in this study were unique and patient-specific.

Differentiation of iPS cells into iPS-ECs from non-diabetic volunteers and diabetic donors produces phenotypically-comparable cells

Upon iPS cell differentiation to iPS-ECs, flow cytometry demonstrated that before CD144 MACS selection up to $57.2 \%$ of iPS-ECs were CD144+ (Supplementary Figure S1); up to $67.2 \%$ of cells were CD144+ in the DME samples (Supplementary Figure S2). iPS-ECs from diabetic patients and non-diabetic volunteers displayed loss of expression of pluripotent markers such as OCT4, LIN28 and NANOG and upregulation of EC markers such as CD144, CD31 and VEGFR2 (Figures $3 \mathrm{~A}-\mathrm{B}$ and $4 \mathrm{~A}-\mathrm{B}$ ) at the mRNA level, confirming differentiation towards the vascular lineage. Further characterisation by immunoblotting (Figures $3 \mathrm{C}$ and $4 \mathrm{C}$ ) also confirmed that cells switched off the expression of the pluripotency marker TRA-1-60, but highly expressed EC-specific markers such as CD144. There was no difference in EC gene expression and immunophenotype among iPS-EC groups (Figures 3 and 4) nor was there a difference, based on immunofluorescent staining, on adherens and tight junction integrity or VEGFR2 expression (Figures 3D and 4D). These resuts clearly demonstrate that iPS-ECs were successfully generated and expanded from all groups (non-diabetic donors and diabetic patients with and without DME).

iPS-ECs from non-diabetic volunteers and diabetic donors, including DME "responders" and "non-responders", have similar phenotypical characteristics but different permeability responses.

iPS-ECs from non-diabetic donors, diabetic patients with mild DR, DME "responders" and "non-responders" have displayed similar functional capacities. The migration capacity of iPSECs from non-diabetic and diabetic or "responders" and "non-responders" was not significantly different when assessed using a wound healing assay or scratch assay (Figures 5A-B, D-E). In a similar manner, no significant difference in their proliferation capacity was observed (Figure $5 \mathrm{C}$ and $\mathrm{F}$ ). One of the main events in DME is the excessive permeability within the normally "tight" retinal microvasculature. Therefore, EC permeability was tested in this study following stimulation with VEGF. Significant differences in VEGF-induced cell 
permeabilty were identified when comparing diabetic patients and non-diabetic volunteers (Figure 6A-B) and when comparing DME "responders" with "non-responders" (Figure 6C-D). In particular, iPS-ECs from "non-responders" had significantly higher increased cell impedance (represented as cell index) shortly after VEGF addition (Figure 6C-D). After 30 and even 90 minutes following VEGF exposure, iPS-ECs derived from DME groups showed decreased cell impedance (i.e. increased permeability) and none showed a recovery pattern like that observed in healthy volunteers in Figure 6A-B. iPS-ECs from "non-responders" showed significantly lower impedance compared to "responders" (Figure 6C-D). We have validated these data by performing further permeability experiments using 3 more iPS-ECs lines (total $\mathrm{n}=6$ for each group), which confirmed increased FITC-Dextran leakage in "non-responders" (Supplementary Figure S4). The relative percent increase in FITC-Dextran diffusion observed in "non-responders" validated the cell impedance data mentioned above. Importantly, we compared the barrier integrity of our iPS-ECs to that of primary human retinal microvascular endothelial cells (HRMECs) using xCELLigence. The results showed that the iPS-ECs were able to reach comparable levels of impedance of cell index (proportional to barrier integrity) to that of HRMECs (data not shown). In addition, the obtained cell index values were comparable or higher to those obtained by other groups working with human brain microvascular ECs [21]. We can therefore confirm that our cells' barrier integrity matches that of central nervous system ECs.

Phosphorylation cascade upon VEGF stimulation reveals differences between DME "responders" and "non-responders".

Given that significant differences in permeability responses of iPS-ECs from DME "responders" and "non-responders" were identified, we aimed to shed light into the mechanism by which this might happen. VEGF induction of EC permeability starts with the binding of VEGF to VEGFR2, which then is phosphorylated triggering a complex signalling cascade that culminates in opening of adherens and tight junctions between ECs. VEGFR2 phosphorylation was assessed by stimulating iPS-ECs from "responders" and "nonresponders" with VEGF for 5 and 20 minutes. Untreated iPS-ECs from both groups were used as control (0 minutes of VEGF stimulation). Interestingly, western blots have shown an increased phosphorylation of VEGFR2 in samples from "non-responders" at the 5-minute time 
point, when compared to "responders" (Figures 7A-B). As a read-out for downstream VEGFR2 signalling, phosphorylation of eNOS, which has a role in the regulation of endothelial permeability, was evaluated and there was no significant difference between groups (Figure 7A-B). These results indicate that ECs generated from "non-responders" have an increased phosphorylation pattern from VEGFR2.

\section{Differential mRNA expression between DME "responders" over "non-responders" and the role of neuronal pentraxin 2 (NPTX2)}

In order to obtain a more thorough analysis into the intrinsic differences between iPS-ECs from "responders" and "non-responders" RNA-Sequencing was performed, which disclosed 13 differentially expressed genes (DEGs) ( $p$-value $<0.05$ ) between "responders" and "nonresponders" (Figure $8 \mathrm{~A}$ and $\mathrm{B}$ ). Further analysis revealed that these genes were involved in processes such as regeneration, repair and cell survival (those showing a higher expression levels in "responders") and inflammation or susceptibility to diabetes (those showing a higher expression in "non-responders"). These 13 DEGs, of which six were upregulated in iPS-ECs from "responders" (Figure 8C) and 7 were upregulated in iPS-ECs from "non-responders" (Figure 8D), could cluster biological replicates of similar samples together. Among these DEGs, NPTX2 was of particular note as it has been previously identified as a biomarker of edema in gliomas [22] and is also expressed in the retina [23]. Strikingly, NPTX2 is also related to CReactive protein (CRP), one of the inflammatory biomarkers involved in the pathogenesis of DR [24], which could mean that it is contributing to inflammation in the non-responder microvasculature. The upregulation of NPTX2 was further assessed in iPS-ECs from "nonresponders" using qRT-PCR (Figure 8E). iPS-ECs from "non-responders" expressed higher NPTX2 than those from "responders". Since phosphorylated VEGFR2 was also higher in "nonresponders" after 5 minutes VEGF exposure (Figure 7A), we hypothesized that there may be a direct correlation with NPTX2. As such, knockdown of NPTX2 (Figure 8F) for 72 hours by lentiviral gene transfer in "non-responders" showed significantly decreased phosphorylation levels of VEGFR2 at the 5 minute time-point compared to its corresponding 5 -minute shNT control (Figures 8F-H). In addition, given that NPTX2 is higher in "non-responders", VEGF levels might directly or indirectly regulate the levels of NPTX2, as it is indicated in Figure 8F. Further NPTX2 silencing experiments based on 3 additional patient specific iPS-ECs "nonresponder" lines showed a pattern of relative percent decrease in diffusion of FITC-Dextran 
(Supplementary Figure S5) in shNPTX2 compared to shNT. This could suggest NPTX2 silencing might serve as a target in improving leakage in "non-responders".

\section{DISCUSSION}

The current study shows that only a proportion of patients with DME achieve full resolution of their macular fluid during the first year of treatment with anti-VEGF therapy. Having first characterised the phenotype and responsiveness of patients with DME to anti-VEGF therapy, we subsequently demonstrated functional differences in their iPS-ECs in vitro, which is a novel discovery. Importantly, the ability to separate DME "full responders" and "non-responders" to anti-VEGFs in a laboratory setting has created a unique platform for deeper patient stratification and, importantly, for the understanding of pathogenic mechanisms of disease and response to anti-VEGF treatment.

Previous studies have used CRT on SD-OCT to determine the response to anti-VEGF therapy. There is, however, no consensus on a definition of "responder" and "non-responder". Thus, Lee et al. [13] considered a response when there was a reduction in CRT of $>50 \mu \mathrm{m}$ after three consecutive anti-VEGF injections, whereas Dabir et al. [14] when the reduction was $>10 \%$ after two consecutive injections. Pieramici et al. [11], using data from RISE and RIDE, proposed the definition of "immediate" and "delayed" responders. Based on data from the DRCR.net Protocol I, where the terms "strong early" and "limited early" responders were used, there was a reduction of CRT on SD-OCT after the same loading dose [12]. The measure of CRT on SD-OCT, although helpful, may not be the best way to determine the response to anti-VEGF therapy given the great range of normal values among individuals, variability based on the instruments used to obtain CRT values, and the fact that DME may still be present in the context of a "normal" CRT value in patients in whom neurodegeneration may have occurred. We considered "full responders" those individuals who achieved a fully dry macula at any time during the first year of treatment. In our cohort, the macula of "full responders" dried after a median time of 7 months (range 1-12 months). Waiting longer than a year to determine the response to treatment and to consider switching to a different therapeutic alternative may compromise functional recovery. In only a small percentage of patients in our study the macula dried fully after a year of adequate treatment with anti-VEGFs. Although the cost-effectiveness of anti-VEGF therapies licenced for the treatment of DME and currently in used in clinical practice (Ranibizumab and Aflibercept) has been demonstrated [25], and 
respectively [26], data used to evaluate their clinical and cost-effectiveness was that obtained in Randomised Clinical Trials (RCTs). It is acknowledged now that RCT results, however, are often not reproduced in clinical practice [27]. Thus, it remains to be elucidated if the costeffectiveness of anti-VEGF therapies remains over appropriate thresholds in real-life clinical settings.

In this study, we used iPS cell technology to make an in vitro model of the retinal microvasculature of patients with DME. In most previous diabetes-related studies, the main source for iPS cell generation was dermal fibroblasts [28]. These are easily maintained and reprogrammed but must be obtained through a skin biopsy [29], a procedure which carries ethical challenges, especially for diabetic patients who suffer from delayed healing [30]. In contrast, we demonstrated that iPS cells can be obtained successfully from blood MNCs using a non-integrating episomal-based transfection and avoiding the long-term incorporation of reprogramming factors into chromosomal DNA.

Patient-specific iPS cells were differentiated into vascular ECs as they are critical components of the retinal neurovascular unit (NVU) and their dysfunction is intimately linked to loss of integrity of the inner BRB. Primary ECs have been widely used to study endothelial permeability and molecular pathways in vitro [31,32], although these are often obtained from animals or post-mortem donors and the human disease-related information they can provide is limited. In contrast, iPS-ECs as generated in this study provided a human disease model which, importantly, is specifically linked to individual patients who have been clinically phenotyped. In addition, since iPS cells can retain information from the donor cells in terms of epigenetic memory [33], this can predispose the subsequent differentiated cells into exhibiting a donor-related epigenetic signature. Recently, it was reported that vascular progenitors generated from tankyrase inhibitor-regulated naïve diabetic human iPS cells revascularized the ischemic retina suggesting erasure of dysfunctional epigenetic donor-cell memory [34]. This phenomenon has been described in other cells such as cardiomyocytes, where treatment with high glucose induces "metabolic memory" [35].

It is acknowledged that iPS-ECs, as generated herein, are not retina-specific. However, one of the key characteristics of retinal microvascular endothelial cells is their low permeability due to their tight barrier properties. Even if the iPS-ECs used in this study are not retinaspecific, their barrier properties, checked as cell impedance level, are equivalent to those of human retinal microvascular endothelial cells and human brain microvascular endothelial 
cells [36]. Furthermore, although ECs are key to the pathogenesis of DME, they are not the only cells responsible for the maintenance of the inner BRB, with integrity of the NVU also being influenced by pericytes, Müller glia, neurons and immune cells. In contrast, the outer BRB is formed by the retinal pigment epithelium (RPE), which is also dysfunctional in diabetes [37]. Considering that iPS cells can be differentiated into any type of cell in the body, studies in our lab are currently underway to generate retinal-specific cells such as RPE and Müller glia to evaluate their role in DME in a patient-specific manner [38]. Co-cultures of iPS-ECs with other iPS-derived cells which form the NVU [39] would also provide a model closer to that of the living retina [40].

In this study, iPS-ECs from non-diabetic donors, diabetic patients with mild DR, DME "responders" and "non-responders" displayed similar functional capacities. The migration capacity of iPS-ECs from non-diabetic and diabetic or "responders" and "non-responders" was not significantly different. In a similar manner, no significant difference in their proliferation capacity was observed. In particular, when iPS-ECs from "responders" and "non-responders" were compared, there were no significant differences in their morphology, expression of EC markers and most functional capacities according to the tests performed. This confirms that iPS cells from diabetic patients can be efficiently differentiated into functional ECs. Because in DME there is excessive EC permeability, we assessed the barrier function capacity of iPSECs and their permeability response to VEGF in vitro. When analysing iPS-ECs from "responders" and "non-responders" we observed that these cells did not recover their initial EC barrier function even 90 minutes following VEGF stimulation. Interestingly, our results showed that iPS-ECs from "non-responders" had a higher permeability response to VEGF when compared to "responders", suggesting that ECs from "non-responders" are more sensitive to the effects of VEGF. This suggests that ECs from "non-responders" may be activated even with very low levels of intraocular VEGF that may remain despite of the blockade achieved by anti-VEGFs. Since the VEGF-mediated increase of EC permeability is driven by a complex phosphorylation cascade [41], we assessed the phosphorylation status of VEGFR2, eNOS and other 46 kinases (Figure 7, Supplementary Figure S3) after VEGF stimulation in iPS-ECs from "responders" and "non-responders", focusing on several VEGFrelated pathways. Results showed that VEGFR2 was differentially phosphorylated between these groups, with "non-responders" demonstrating greater phosphorylation of this receptor than "responders". These results are in agreement with those from the permeability studies 
reinforcing the hypothesis that "non-responders" may be highly sensitive to the effects induced by VEGF. If the "non-responder" receptors were to be more sensitive to VEGF and, therefore, had a lower threshold for VEGFR2 activation, even low levels of VEGF that may persist following anti-VEGF blockade in vivo would be able to maintain vascular leakage through VEGFR2 signalling with persistence of DME despite treatment.

In order to deepen the understanding of the differences between these two groups, RNA-Seq of their iPS-ECs was performed and a differential gene expression profile was observed between "responders" and "non-responders". A total of 13 DEGs were identified to be significantly expressed with criteria of adjusted $p$ value $<0.05$ and absolute log 2 fold change greater than 1. One of the genes found to be upregulated in "non-responders" was NPTX2. NPTX2 has been identified as a potential biomarker of edema in gliomas [22] and also appears to be related to C-Reactive protein, one of the inflammatory biomarkers involved in the pathogenesis of DR [42]. There is, thus, a possibility that higher expression of this protein in the endothelium of "non-responders" could be driving edema through a VEGF parallel pathway, which could be an additional reason why these patients fail to respond to VEGF inhibition. Silencing NPTX2 in "non-responders" diminished the effect of VEGF in the phosphorylation of VEGFR2, suggesting a possible effect in reducing inflammation-related events. Furthermore, NPTX2 silencing reduced the relative FITC-Dextran leakage in "nonresponders" showcasing that targeting this gene could play a role in correcting barrier integrity. Studies have shown that abnormal upregulation in NPTX2 expression was linked to the proliferation and metastasis of cancers such as clear cell renal cell carcinoma through the activation of the Wnt/ $\beta$-catenin signaling pathway. NPTX2 was shown to interact with frizzled class receptor 6 (FZD6) to induce $\beta$-catenin nuclear translocation, with an ensuing decrease in the expression of E-cadherin [43] and an increase in the expression of MYC, snail, Ncadherin and cyclin D1, which induces VEGF production [44].

The strengths of this study include the identification of consecutive patients through an electronic database; the masking of outcome assessors (i.e. the researcher grading the OCT scans and very importantly the scientists undertaking iPS-EC experiments and analysing their results were masked to the treatment response observed in clinic); the evaluation of all OCT scans obtained throughout the whole year of treatment in order to determine the type of treatment response observed in all patients included in the cohort; and the robust techniques used to obtain patient-specific iPS and iPS-ECs. One of the limitations is the small sample size 
$(n=6)$ used for the in vitro studies. However, since these patient specific iPS-ECs have been generated based on a very well-characterised clinical cohort they allowed robust differences to be revealed and conclusions to be made. The present study sets the foundation to identify key pathophysiological differences between "full responders" and "non-responders" to antiVEGF treatment among DME patients, which may have potential clinical relevance, and also provide scope for new drug development.

\section{Conclusions}

By generating iPS-ECs from non-diabetic volunteers, people with diabetes but no DME and people with DME who are "full responders" or "non-responders" to anti-VEGF, we found increased cell permeability in diabetics compared to controls following stimulation with VEGF in vitro, an observation which was exaggerated in "non-responders" when compared to "full responders". Additionally, phosphorylation of key cellular proteins following VEGF, including VEGFR2, and gene expression profiles, such as NPTX2, differed between "full responders" and "non-responders" allowing for future possible identification of responsive patients to treatment. 


\section{Acknowledgments}

We thank Eimear Byrne for her expert advice on the FITC-Dextran experiments.

\section{Author contributions}

*M.V.G., *M.E., and *S.K. conception and design, collection and/or assembly of data, data analysis and interpretation, manuscript writing; S.F., S.S., G.V., collection and/or assembly of data; H.N. RNA-seq data analysis and interpretation, D.J.G., provision of study material, final approval of manuscript; A.W.S., N.L., A.M.: conception and design of the study, collection and/or assembly of data, data analysis and interpretation, manuscript writing, financial support, final approval of manuscript.

\section{Sources of Funding}

This work was supported by grants from the MRC, BBSRC, and British Heart Foundation.

\section{Conflict of interest statement}

The authors indicate no potential conflicts of interest.

\section{Guarantor statement}

"Dr Andriana Margariti and Prof Noemi Lois are the guarantors of this work and, as such, had full access to all the data in the study and take responsibility for the integrity of the data and the accuracy of the data analysis." 


\section{References}

1. Lee, R., T.Y. Wong, and C. Sabanayagam, Epidemiology of diabetic retinopathy, diabetic macular edema and related vision loss. Eye Vis (Lond), 2015. 2: p. 17.

2. Cunha-Vaz, J., J.R. Faria de Abreu, and A.J. Campos, Early breakdown of the bloodretinal barrier in diabetes. Br J Ophthalmol, 1975. 59(11): p. 649-56.

3. Stitt, A.W., et al., The progress in understanding and treatment of diabetic retinopathy. Prog Retin Eye Res, 2016. 51: p. 156-86.

4. Funatsu, H., et al., Vitreous levels of pigment epithelium-derived factor and vascular endothelial growth factor are related to diabetic macular edema. Ophthalmology, 2006. 113(2): p. 294-301.

5. Diaz-Coranguez, M., C. Ramos, and D.A. Antonetti, The inner blood-retinal barrier: Cellular basis and development. Vision Res, 2017. 139: p. 123-137.

6. Elman, M.J., et al., Randomized trial evaluating ranibizumab plus prompt or deferred laser or triamcinolone plus prompt laser for diabetic macular edema. Ophthalmology, 2010. 117(6): p. 1064-1077 e35.

7. Nguyen, Q.D., et al., Ranibizumab for diabetic macular edema: results from 2 phase III randomized trials: RISE and RIDE. Ophthalmology, 2012. 119(4): p. 789-801.

8. Mitchell, P., et al., The RESTORE study: ranibizumab monotherapy or combined with laser versus laser monotherapy for diabetic macular edema. Ophthalmology, 2011. 118(4): p. 615-25.

9. Korobelnik, J.F., et al., Intravitreal aflibercept for diabetic macular edema. Ophthalmology, 2014. 121(11): p. 2247-54.

10. Wells, J.A., et al., Aflibercept, bevacizumab, or ranibizumab for diabetic macular edema. N Engl J Med, 2015. 372(13): p. 1193-203.

11. Pieramici, D.J., et al., Visual and Anatomic Outcomes in Patients with Diabetic Macular Edema with Limited Initial Anatomic Response to Ranibizumab in RIDE and RISE. Ophthalmology, 2016. 123(6): p. 1345-50.

12. Dugel, P.U., et al., ASSOCIATION BETWEEN EARLY ANATOMIC RESPONSE TO ANTIVASCULAR ENDOTHELIAL GROWTH FACTOR THERAPY AND LONG-TERM OUTCOME IN DIABETIC MACULAR EDEMA: An Independent Analysis of Protocol i Study Data. Retina, 2019. 39(1): p. 88-97.

13. Lee, J., et al., Optical Coherence Tomography Angiography of DME and Its Association with Anti-VEGF Treatment Response. Ophthalmology, 2016. 123(11): p. 2368-2375.

14. Dabir, S.S., et al., Differential systemic gene expression profile in patients with diabetic macular edema: responders versus nonresponders to standard treatment. Indian J Ophthalmol, 2014. 62(1): p. 66-73.

15. Elman, M.J., et al., Intravitreal Ranibizumab for diabetic macular edema with prompt versus deferred laser treatment: 5-year randomized trial results. Ophthalmology, 2015. 122(2): p. 375-81.

16. Sachdeva, M.M., et al., Endophthalmitis following intravitreal injection of anti-VEGF agents: long-term outcomes and the identification of unusual micro-organisms. Journal of ophthalmic inflammation and infection, 2016. 6(1): p. 2-2.

17. Vila-Gonzalez, M., et al., Enhanced Function of Induced Pluripotent Stem Cell-Derived Endothelial Cells Through ESM1 Signaling. Stem Cells, 2019. 37(2): p. 226-239.

18. Margariti, A., et al., Histone deacetylase 7 controls endothelial cell growth through modulation of beta-catenin. Circ Res, 2010. 106(7): p. 1202-11. 
19. Margariti, A., et al., Direct reprogramming of fibroblasts into endothelial cells capable of angiogenesis and reendothelialization in tissue-engineered vessels. Proc Natl Acad Sci U S A, 2012. 109(34): p. 13793-8.

20. Cochrane, A., et al., Quaking Is a Key Regulator of Endothelial Cell Differentiation, Neovascularization, and Angiogenesis. Stem Cells, 2017. 35(4): p. 952-966.

21. Wiltshire, R., et al., Regulation of human cerebro-microvascular endothelial basolateral adhesion and barrier function by S1P through dual involvement of S1P1 and S1P2 receptors. Scientific Reports, 2016. 6(1): p. 19814.

22. Carlson, M.R., et al., Relationship between survival and edema in malignant gliomas: role of vascular endothelial growth factor and neuronal pentraxin 2. Clin Cancer Res, 2007. 13(9): p. 2592-8.

23. Bjartmar, L., et al., Neuronal pentraxins mediate synaptic refinement in the developing visual system. J Neurosci, 2006. 26(23): p. 6269-81.

24. Nowak, M., et al., Antioxidant potential, paraoxonase 1, ceruloplasmin activity and Creactive protein concentration in diabetic retinopathy. Clin Exp Med, 2010. 10(3): p. 185-92.

25. T.N.I.f.H.a.C. Ranibizumab for treating diabetic macular oedema. 2013; Available from: https://www.nice.org.uk/guidance/ta274.

26. T.N.I.f.H.a.C. Aflibercept for treating diabetic macular oedema. 2015; Available from: https://www.nice.org.uk/guidance/ta346.

27. Denniston, A.K., et al., United Kingdom Diabetic Retinopathy Electronic Medical Record (UK DR EMR) Users Group: report 4, real-world data on the impact of deprivation on the presentation of diabetic eye disease at hospital services. The British journal of ophthalmology, 2019. 103(6): p. 837-843.

28. Kondo, Y., et al., iPSC technology-based regenerative therapy for diabetes. J Diabetes Investig, 2018. 9(2): p. 234-243.

29. Vangipuram, M., et al., Skin punch biopsy explant culture for derivation of primary human fibroblasts. Journal of visualized experiments : JoVE, 2013(77): p. e3779e3779.

30. Dangwal, S., et al., Impairment of Wound Healing in Patients With Type 2 Diabetes Mellitus Influences Circulating MicroRNA Patterns via Inflammatory Cytokines. Arterioscler Thromb Vasc Biol, 2015. 35(6): p. 1480-8.

31. van Dijk, I.A., et al., Human salivary peptide histatin-1 stimulates epithelial and endothelial cell adhesion and barrier function. FASEB J, 2017. 31(9): p. 3922-3933.

32. Imai, A., et al., Adrenomedullin Suppresses Vascular Endothelial Growth FactorInduced Vascular Hyperpermeability and Inflammation in Retinopathy. Am J Pathol, 2017. 187(5): p. 999-1015.

33. Kim, K., et al., Epigenetic memory in induced pluripotent stem cells. Nature, 2010. 467(7313): p. 285-90.

34. Park, T.S., et al., Vascular progenitors generated from tankyrase inhibitor-regulated naïve diabetic human iPSC potentiate efficient revascularization of ischemic retina. Nature Communications, 2020. 11(1): p. 1195.

35. Yu, X.Y., et al., High levels of glucose induce "metabolic memory" in cardiomyocyte via epigenetic histone H3 lysine 9 methylation. Mol Biol Rep, 2012. 39(9): p. 8891-8.

36. Wiltshire, R., et al., Regulation of human cerebro-microvascular endothelial basolateral adhesion and barrier function by S1P through dual involvement of S1P1 and S1P2 receptors. Sci Rep, 2016. 6: p. 19814. 
37. Duh, E.J., J.K. Sun, and A.W. Stitt, Diabetic retinopathy: current understanding, mechanisms, and treatment strategies. JCl Insight, 2017. 2(14).

38. Yang, J., et al., Induced Pluripotent Stem Cells and Outer Retinal Disease. Stem cells international, 2016. 2016: p. 2850873-2850873.

39. Orlova, V.V., et al., Functionality of endothelial cells and pericytes from human pluripotent stem cells demonstrated in cultured vascular plexus and zebrafish xenografts. Arterioscler Thromb Vasc Biol, 2014. 34(1): p. 177-86.

40. Hollmann, E.K., et al., Accelerated differentiation of human induced pluripotent stem cells to blood-brain barrier endothelial cells. Fluids Barriers CNS, 2017. 14(1): p. 9.

41. Weis, S.M. and D.A. Cheresh, Pathophysiological consequences of VEGF-induced vascular permeability. Nature, 2005. 437(7058): p. 497-504.

42. Lim, L.S., et al., C-reactive protein, body mass index, and diabetic retinopathy. Invest Ophthalmol Vis Sci, 2010. 51(9): p. 4458-63.

43. $\mathrm{Xu}, \mathrm{C}$., et al., NPTX2 promotes colorectal cancer growth and liver metastasis by the activation of the canonical Wnt/B-catenin pathway via FZD6. Cell Death \& Disease, 2019. 10(3): p. 217.

44. Tsai, H.L., et al., Co-existence of cyclin D1 and vascular endothelial growth factor protein expression is a poor prognostic factor for UICC stage I-III colorectal cancer patients after curative resection. J Surg Oncol, 2013. 107(2): p. 148-54. 


\section{Figure Legends}

Figure 1. (A) Colour fundus photographs of the right eye obtained from an individual with no retinal disease (top, left) and from a patient with diabetic macular edema (DME) (top, right). Hard exudation is seen involving the macula and extending to the fovea (black arrow). (B) Spectral domain optical coherence tomography (SD-OCT) scans obtained at baseline (left, middle) and at a time during the first year when the retina dried (right, middle) in a "full responder"; and at baseline (left, bottom) and at the end of the year following repeated treatments (right, bottom) in a "non-responder". Intraretinal (white arrow) and subretinal fluid (white arrow head) are observed at presentation in the "full responder" with complete resolution following treatment and recovery of a near normal macular structure (middle panels). Intraretinal fluid (white arrow) is seen in a "non-responder" with minimal change throughout the first year of treatment (bottom panels).

Figure 2. Generation and characterisation of iPS cells from non-diabetic donors, diabetic patients with mild DR, DME Responders and DME Non-Responders. (A) qPCR analysis shows erythroid markers are no longer present in reprogrammed cells. (B) There are similar mRNA levels of pluripotent markers in all groups, when compared to MNCs (somatic cells of origin). (Data are means \pm SEM $\left.(n=3),{ }^{*} p<0.05,{ }^{* *} p<0.01\right)$. (C) Pluripotent markers are also expressed at a protein level in all groups. (D) Immunofluorescence staining confirms the characterisation of iPS cells in all groups. Scale bars are $50 \mu \mathrm{m}$ (white), $100 \mu \mathrm{m}$ (yellow) or $200 \mu \mathrm{m}$ (orange).

Figure 3. iPS-ECs differentiation and characterisation. iPS-ECs were successfully generated from diabetic patients and from non-diabetic donors. (A) qPCR analysis shows that iPS-ECs from both groups have switched off the expression levels of pluripotent markers such as OCT4, LIN28, NANOG and (B) they highly expressed endothelial cells specific markers such as CD144, CD31, VEGFR2, (Data are means $\pm \operatorname{SEM}(n=3),{ }^{*} p<0.05,{ }^{* *} p<0.01,{ }^{* * *} p<0.001$ ). (C) These results were also confirmed in the protein levels by western blots. (D) Immunofluorescence staining of endothelial markers confirms the above results and demonstrates that iPS-ECs from both groups were able to form endothelial and adherent junctions. Scale bars are $50 \mu \mathrm{m}$.

Figure 4. iPS-ECs were successfully generated from DME patients (Responders $(R)$ and NonResponders (NR)) in a similar way as iPS-ECs from diabetic patients and from non-diabetic donors. (A) qPCR analysis shows that iPS-ECs from both groups have stopped expressing the pluripotent markers such as OCT4, LIN28, NANOG and (B) they highly expressed endothelial cells specific markers such as CD144, CD31, VEGFR2, (Data are means \pm SEM $(n=3),{ }^{*} p<0.05$, $* * * p<0.001$ ). (C) These results were also confirmed by western blots in the protein level. (D) Immunofluorescence staining of endothelial markers confirms that iPS-ECs from DME patients (Responders (R) and Non-Responders (NR)) were able to form endothelial and adherent junctions. Scale bars are $50 \mu \mathrm{m}$.

Figure 5. iPS-ECs from non-diabetic donors, diabetic patients with mild DR, DME Responders and Non-Responders have similar functional capacities. (A) and (D): Representative images of an in vitro wound healing process at different timepoints. (B) and (E): The migration capacity between iPS-ECs from non-diabetic and diabetic or Responders and Non-Responders is not significantly different. It is measured as area measurement change from baseline in a 
wound healing assay or scratch assay. (Data are means \pm SEM $(n=3)$ ). (C) and (F): iPS-ECs from non-diabetic and diabetic or Responders and Non-Responders show no significant difference in their proliferation capacity. (Data are means \pm SEM $(n=3)$ ).

Figure 6. Permeability response to VEGF stimulation in iPS-ECs. Permeability response of iPSECs in response to VEGF stimulation showed significant differences between non-diabetic donors and diabetic patients and between DME responders and non-responders. (A-B) significant differences in cell permeabilty in response to VEGF were identified when comparing diabetic patients and non-diabetic volunteers, and when comparing (C-D) DME "full responders" with "non-responders". iPS-ECs from DME "non-responders" had significantly higher increased cell impedance shortly after addition of VEGF. (C-D) iPS-ECS from DME "non-responders" showed significantly lower impedance compared to "full responders". (Data are means \pm SEM $(n=3), * * * p<0.001$ ).

Figure 7. Phosphorylation state of proteins in response to VEGF stimulation in iPS-ECs: (A) Western blot analysis of P-VEGFR2 in iPS-ECs from DME responders (R) and non-responders (NR) at different time points after VEGF treatment. $\beta$-actin was used as a loading control. (B) Quantification of the western blot. P-VEGFR2 (Phospho-VEGFR2) signal at 5 minutes is double in non-responders compared to responders while P-eNOS shows no significant difference between the two groups. (Data are means $\left.\pm \operatorname{SEM}(n=3),{ }^{*} p<0.05\right)$.

Figure 8. Differences in RNA expression between DME responders and non-responders and the role of NPTX2. (A) Volcano plot showing differentially expressed genes (DEGs) between responder versus non-responder. The $x$-axis represents the log 2 fold change, while the $y$-axis corresponds to $-\log 10$ Adjusted P-Value with the significance cut-off 1.3 represents by horizontal dotted line (equal to adjusted $p$-value 0.05 ). Every red point in the plot corresponds to a significant DEG with a fold change greater than +1 for up-regulated genes (right vertical dotted line) and less than -1 for down-regulated genes (left vertical dotted line) for responder against non-responder. In particular, NPTX2 is shown by a purple text box. (B) Heat map clustering the samples based on 13 significant DEGs between responder against nonresponder. (C) Relative expression of differential gene expression between responders and non-responders. (D-E) NPTX2 is upregulated in non-responders. (F) NPTX2 knockdown in 72 hours after lentiviral gene transfer in non-responders delays phosphorylation of VEGFR2 at the 5 minute time-point of VEGF exposure. (G-H) Densitometry shows quantification levels of NPTX2 and P-VEGFR2. (Data are means \pm SEM $\left.(n=3), *^{*}<0.05,{ }^{*} p<0.01\right)$. 


\section{Supplementary Table 1}

\begin{tabular}{|c|c|c|c|c|c|c|c|c|c|}
\hline Study ID & $\begin{array}{l}\text { Grou } \\
p\end{array}$ & $\begin{array}{l}\mathrm{Ag} \\
\mathrm{e}\end{array}$ & Sex & $\begin{array}{l}\text { Weight } \\
\text { (kg) }\end{array}$ & $\begin{array}{l}\text { Heigh } \\
t(\mathrm{~cm})\end{array}$ & BMI & $\begin{array}{l}\text { Blood } \\
\text { Pressure } \\
\text { (mmHg) }\end{array}$ & $\begin{array}{l}\text { Diabetes } \\
\text { type }\end{array}$ & $\begin{array}{l}\text { Diabetes } \\
\text { duration }\end{array}$ \\
\hline $\begin{array}{l}\text { STREAM1/0 } \\
03\end{array}$ & $\mathrm{R}$ & 56 & $M$ & 93 & 180 & 28.7 & $135 / 80$ & Type 2 & $\begin{array}{l}10-15 \\
\text { years }\end{array}$ \\
\hline $\begin{array}{l}\text { STREAM1/0 } \\
06\end{array}$ & $\mathrm{R}$ & 47 & $M$ & 94.5 & 181 & 28.8 & $141 / 62$ & Type 1 & $>15$ years \\
\hline $\begin{array}{l}\text { STREAM1/0 } \\
07\end{array}$ & $R$ & 72 & $\mathrm{~F}$ & 63.5 & 147 & 29.4 & $160 / 70$ & Type 2 & $>15$ years \\
\hline $\begin{array}{l}\text { STREAM1/0 } \\
09\end{array}$ & NR & 63 & $M$ & 141 & 164 & 52.4 & $125 / 67$ & Type 2 & $>15$ years \\
\hline $\begin{array}{l}\text { STREAM1/0 } \\
11\end{array}$ & NR & 60 & $\mathrm{~F}$ & 99.1 & 152 & 42.8 & $156 / 61$ & Type 2 & $>15$ years \\
\hline $\begin{array}{l}\text { STREAM1/0 } \\
12\end{array}$ & NR & 89 & $\mathrm{~F}$ & 72.2 & 151 & 31.6 & $134 / 81$ & Type 2 & $5-10$ years \\
\hline
\end{tabular}

Supplementary Table 1: Demographics and characteristics of participants of this study. Legend: R, DME responders; NR, DME Non-Responders 


\section{Supplementary Table 2}

\begin{tabular}{|c|c|c|}
\hline PRIMER & & SEQUENCE \\
\hline \multirow[t]{2}{*}{ CD235 } & Forward & GCCСATCTGATGTAAAACCTCTC \\
\hline & Reverse & TGGTGAACAGATTCTCATTGATCAC \\
\hline \multirow[t]{2}{*}{ CD71 } & Forward & TATCAGGACAGCAACTGGGC \\
\hline & Reverse & CTGCTCGTGCCACTTTGTTC \\
\hline \multirow[t]{2}{*}{ POU5F1 } & Forward & AGAACATGTGTAAGCTGCGG \\
\hline & Reverse & GTTGCCTCTCACTCGGTTC \\
\hline \multirow[t]{2}{*}{ LIN28 } & Forward & GCAGAAGCGCAGATCAAAAG \\
\hline & Reverse & CGGACATGAGGCTACCATATG \\
\hline \multirow[t]{2}{*}{ NANOG } & Forward & GAAATACCTCAGCCTCCAGC \\
\hline & Reverse & GCGTCACACCATTGCTATTC \\
\hline \multirow[t]{2}{*}{ CD144 } & Forward & АААСАССТСАСТТССССАТС \\
\hline & Reverse & ACCTTGCCCACATATTCTCC \\
\hline \multirow[t]{2}{*}{ CD31 } & Forward & TCAGAAGGACAAGGCGATTG \\
\hline & Reverse & GTTATGTTGACCACGATGCTG \\
\hline \multirow[t]{2}{*}{ VEGFR2 } & Forward & ATAGAAGGTGCCCAGGAAAAG \\
\hline & Reverse & GTCTTCAGTTCCCCTCCATTG \\
\hline \multirow[t]{2}{*}{ NPTX2 } & Forward & ССАСТCCGCACAAACTACCT \\
\hline & Reverse & TAGGAGAAGGGGGTGCCAAT \\
\hline
\end{tabular}

Supplementary Table 2: PCR primers and their corresponding sequences 


\section{Supplementary Table 3}

\begin{tabular}{|l|l|}
\hline Treatment Response & Eyes, $\mathbf{n}(\%)$ \\
\hline Full Responders & $39(27.9)$ \\
\hline Partial Responders & $92(65.7)$ \\
\hline Non-Responders & $9(6.4)$ \\
\hline
\end{tabular}

Supplementary Table 3: Anatomical response to anti-VEGF in 100 patients (140 eyes) included in the cohort study. 


\section{Supplementary Table 4}

\begin{tabular}{|c|c|c|c|c|c|}
\hline & $\begin{array}{l}\text { Full Responders } \\
\text { (n= 39) }\end{array}$ & $\begin{array}{l}\text { Partial } \\
\text { Responders } \\
(n=91)\end{array}$ & $\begin{array}{l}\text { Non-Responders } \\
(n=9)\end{array}$ & $\begin{array}{l}\text { Overall } \\
(n=140)\end{array}$ & $\begin{array}{l}\text { p- } \\
\text { value* }\end{array}$ \\
\hline Age & $65(14.2)$ & $65(9.6)$ & 66 (14.9) & $65(11.4)$ & 0.959 \\
\hline $\begin{array}{l}\text { Gender } \\
\text { Male/female }\end{array}$ & $28 / 11$ & $62 / 29$ & $4 / 5$ & $94 / 45$ & 0.398 \\
\hline Type I/II DM & $8 / 31$ & $15 / 76$ & $3 / 6$ & $26 / 113$ & 0.559 \\
\hline BCVA baseline & $59.0(16.7)$ & $59.7(14.8)$ & $63.1(17.5)$ & $59.7(15.4)$ & 0.868 \\
\hline $\begin{array}{l}\text { BCVA } \\
12 \text { months }\end{array}$ & 67.1 (13.9) & $67.7(14.6)$ & $64.2(15.8)$ & $67.3(14.4)$ & 0.853 \\
\hline CRST baseline & $459(140)$ & $491(142)$ & $487(124)$ & $482(140)$ & 0.283 \\
\hline $\begin{array}{l}\text { CRST } \\
12 \text { months }\end{array}$ & $310(87)$ & $327(73)$ & $440(102)$ & $330(84)$ & 0.001 \\
\hline $\begin{array}{l}\text { Anti-VEGF } \\
\text { injections } \\
\text { received by } \\
\text { month } 12\end{array}$ & $6.9(2.2)$ & $7.8(2.0)$ & $7.7(1.2)$ & $7.5(2.0)$ & 0.013 \\
\hline
\end{tabular}

Supplementary Table 4. Demographics and clinical characteristics of "Full Responders", "Partial Responders" and "Non-Responders".

DM = Diabetes Mellitus; BCVA = best corrected visual acuity; CRST = central retinal subfield thickness. In bold, values considered statistically significant. 


\section{Supplementary Table 5}

\begin{tabular}{|r|r|r|r|r|r|r|}
\hline & P003 & P006 & P007 & P009 & P011 & \multicolumn{1}{c|}{ P012 } \\
\hline P003 & & $33.33 \%$ & $26.67 \%$ & $33.33 \%$ & $33.33 \%$ & $33.33 \%$ \\
\hline P006 & $33.33 \%$ & & $30.00 \%$ & $30.00 \%$ & $30.00 \%$ & $30.00 \%$ \\
\hline P007 & $26.67 \%$ & $30.00 \%$ & & $50.00 \%$ & $36.67 \%$ & $26.67 \%$ \\
\hline P009 & $33.33 \%$ & $30.00 \%$ & $50.00 \%$ & & $36.67 \%$ & $16.67 \%$ \\
\hline P011 & $33.33 \%$ & $30.00 \%$ & $36.67 \%$ & $36.67 \%$ & & $33.33 \%$ \\
\hline P012 & $33.33 \%$ & $30.00 \%$ & $26.67 \%$ & $16.67 \%$ & $33.33 \%$ & \\
\hline
\end{tabular}

Supplementary Table 5: The iPS cell lines derived from DME patients are all unique and come from different sources. Short tandem repeat (STR) profiling of these cell lines unveiled that the samples are unique. Comparison between the profiles of each patient shows $<57 \%$ of similarity, which indicates that they come from different sources and therefore there has been no cross contamination.

Cite: ANSI/ATCC ASN-0002-2011. Authentication of Human Cell Lines: Standardization of STR Profiling. ANSI eStandards Store, 2012. 


\section{Supplementary Table 6}
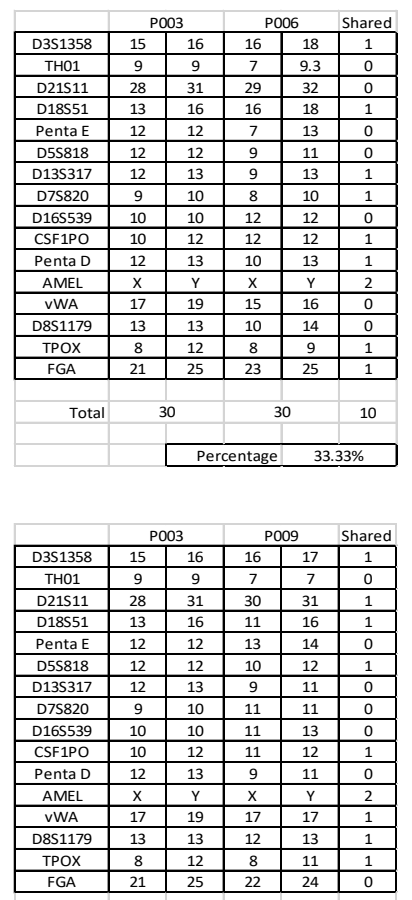

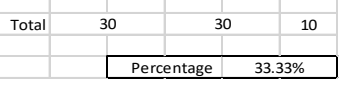

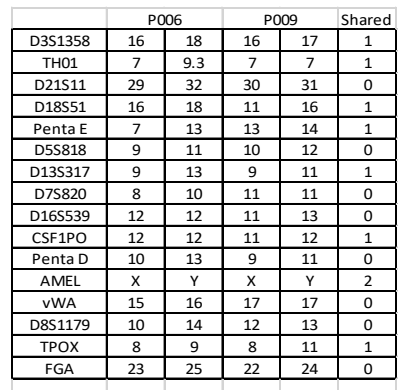

Total 30

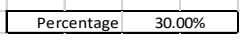

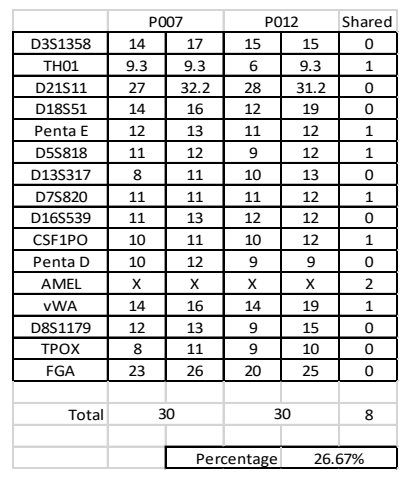

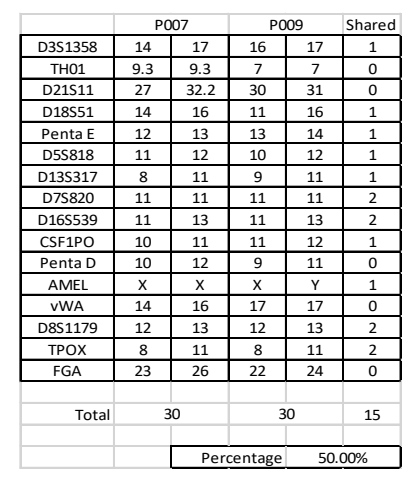
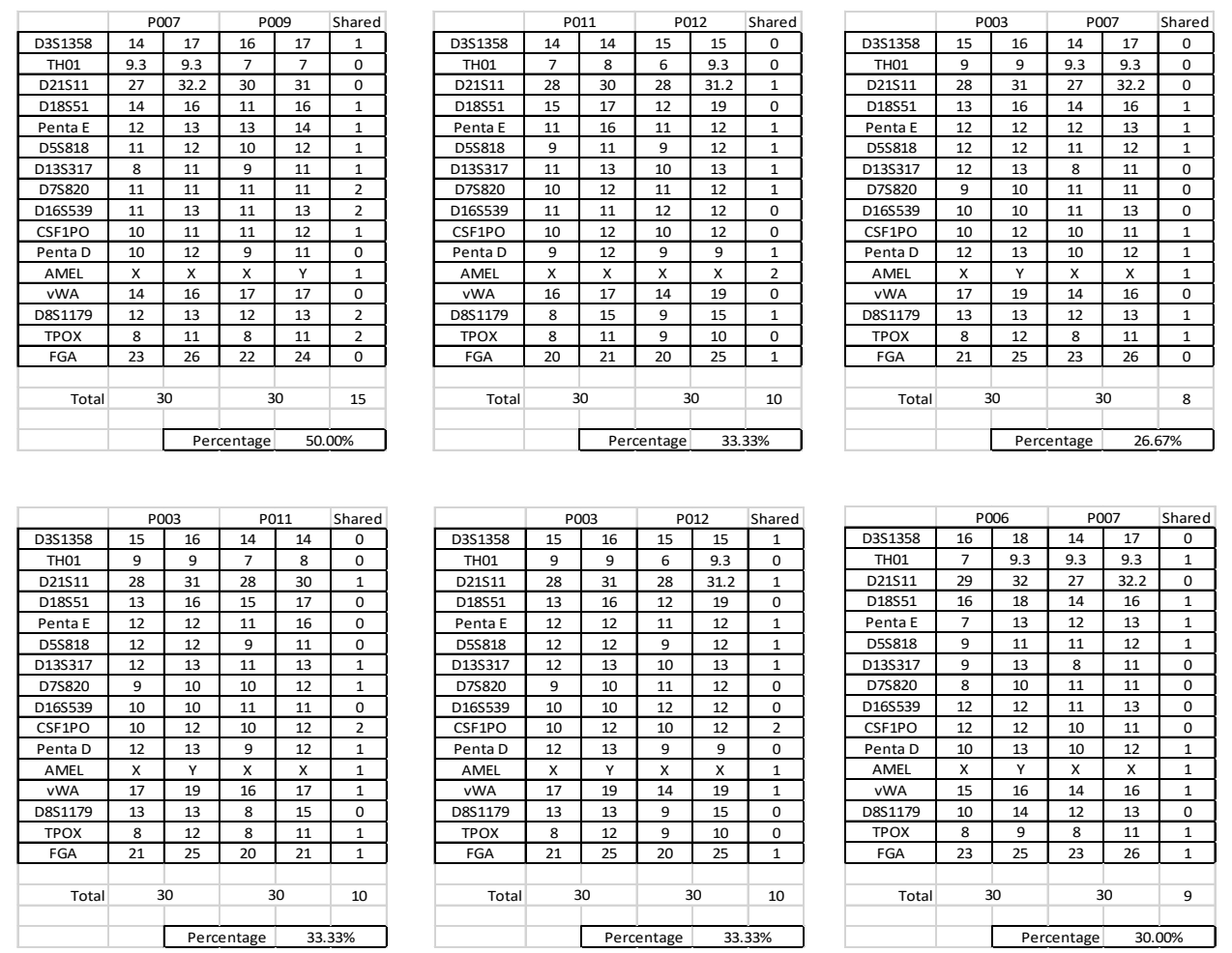

$$
\text { Total } 30
$$

30
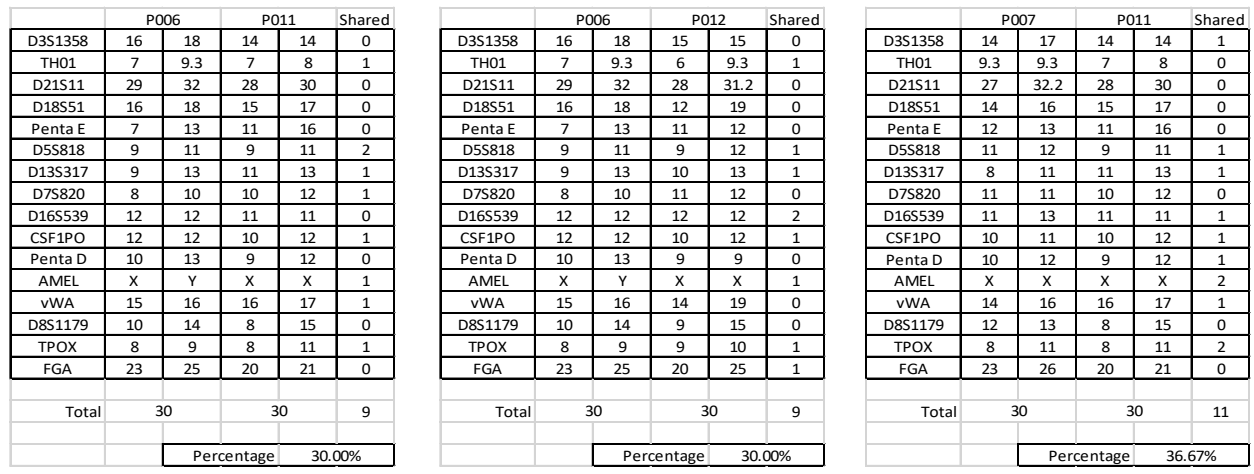

Total $\quad 30$

30

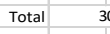

30

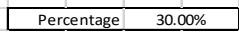

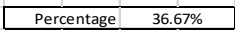
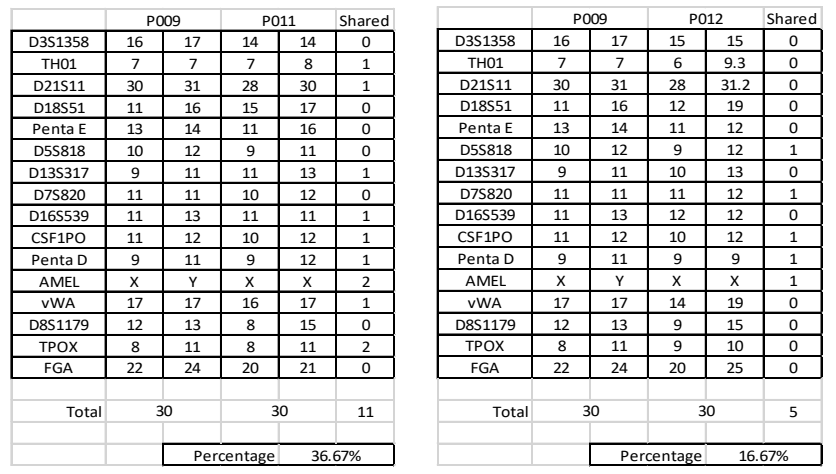

Supplementary Table 6: STR profiling results comparison. The alleles of each cell line were compared side by side with the other lines. The percentage of similarity between lines was always lower or equal to $50 \%$, which means that all cells come from different individuals. 


\section{Supplementary Figure 1}
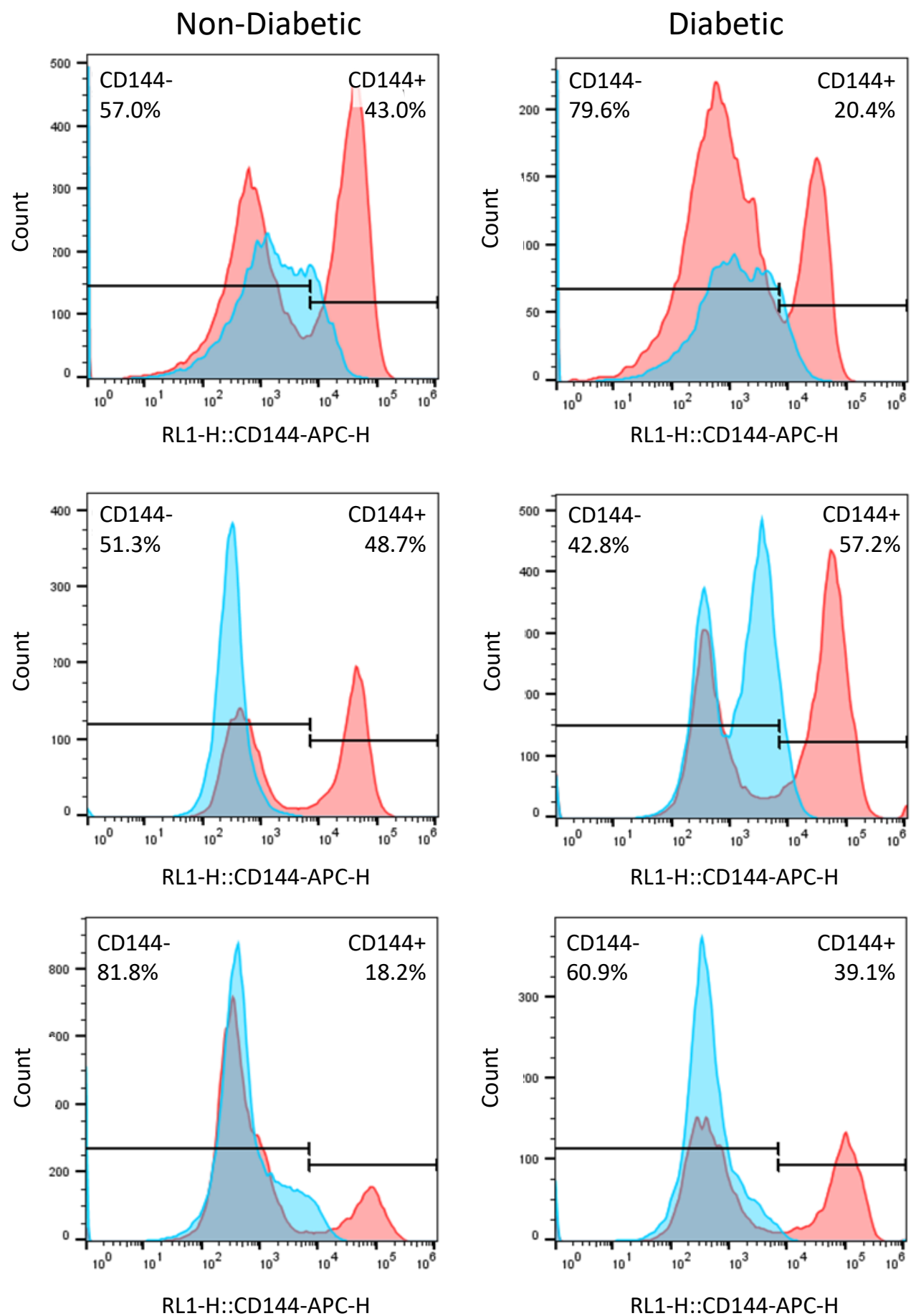

Supplementary Figure 1 : Differentiation efficiency of iPS cells towards endothelial cells. The efficiency is calculated by measuring the percentage of the population that expressed the endothelial marker CD144 on day 6 of differentiation. The levels of CD144+ cells are measured using flow cytometry and compared to an isotype control. The efficiency of the differentiation process in these representative plots of non-diabetic and diabetic cells varies from $18.2 \%$ to $57.2 \%$. 


\section{Supplementary Figure 2}
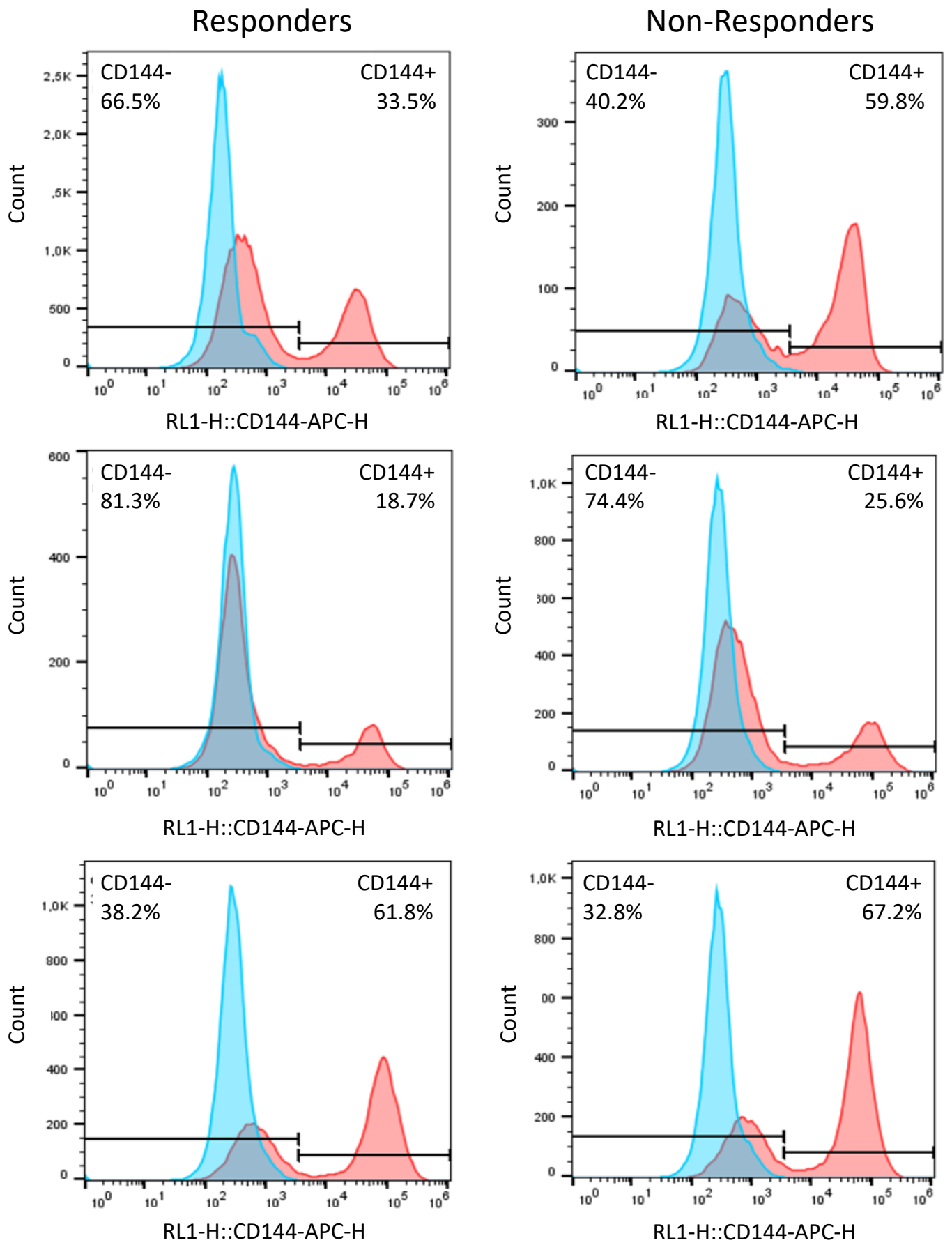

Supplementary Figure 2: Differentiation efficiency of DME iPS cells towards endothelial cells. The efficiency is calculated by measuring the percentage of the population that expressed the endothelial marker CD144 on day 6 of differentiation. The levels of CD144+ cells are measured using flow cytometry and compared to an isotype control. The efficiency of the differentiation process in these representative plots between Responders and Non-Responders varies from $18.7 \%$ to $67.2 \%$. 


\section{Supplementary Figure 3}

A

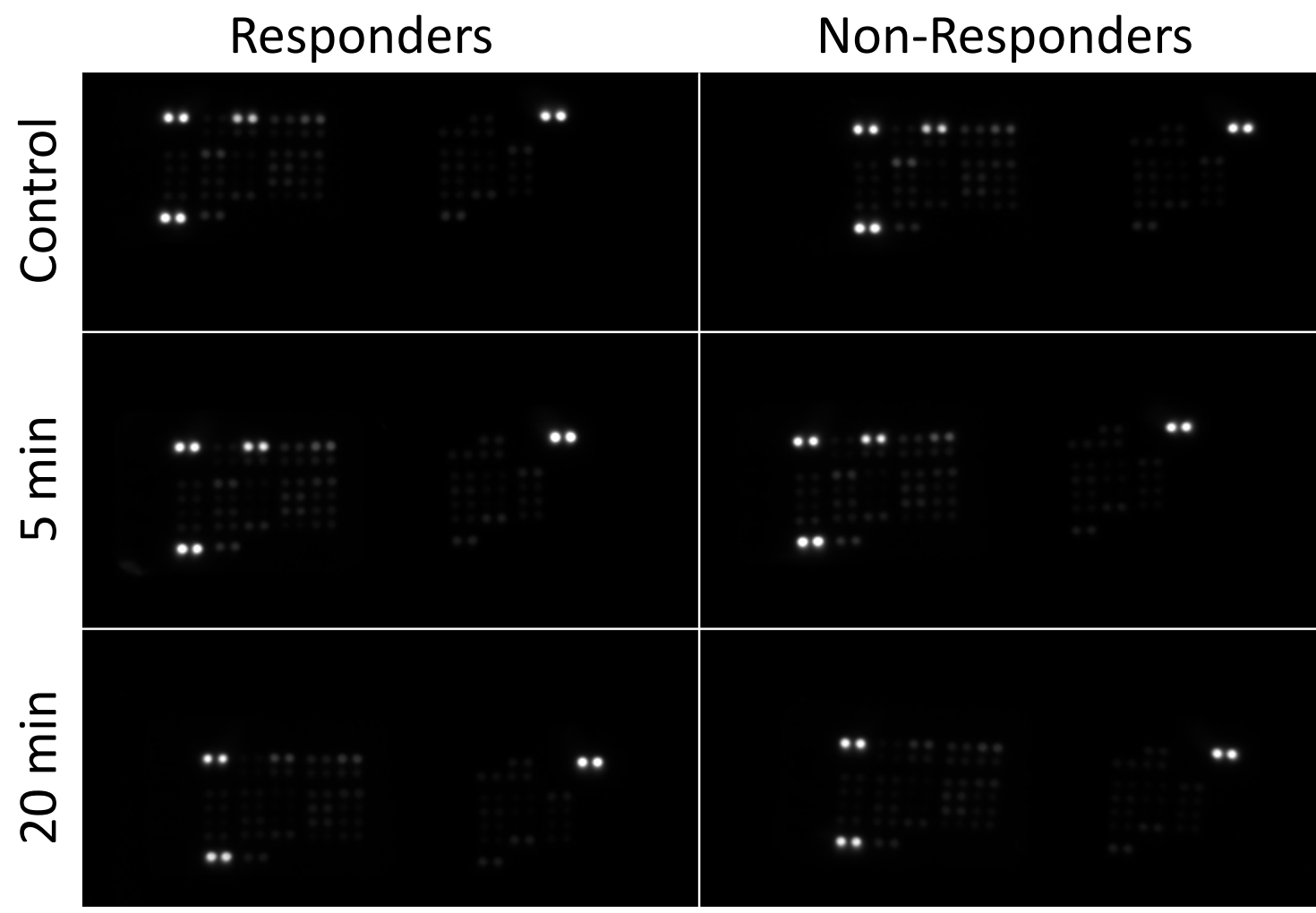

B

$\rightarrow$ Responders - Non-Responders
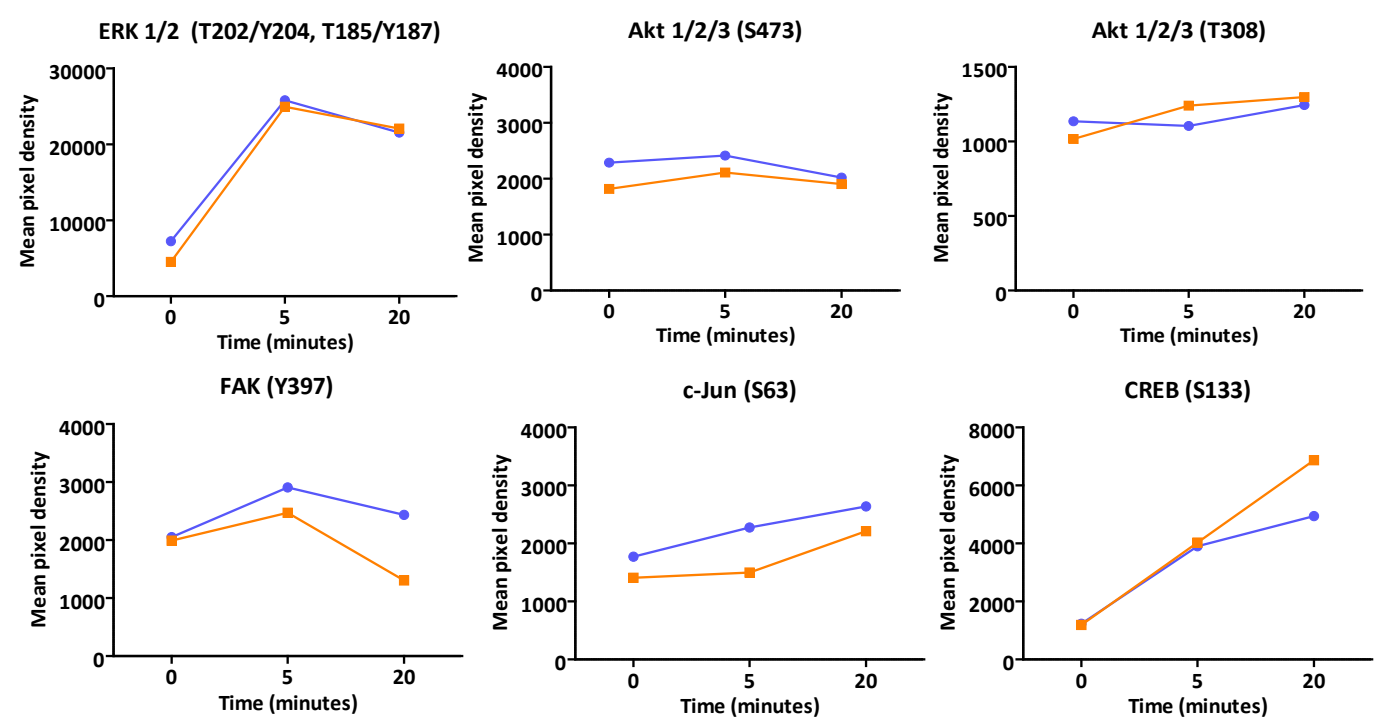

Supplementary Figure 3: Phosphokinase array blotted with protein from iPS-ECs from DME responders or non-responders 5 and 20 minutes after treatment with VEGF. A) In each blot, every dot pair corresponds to a phosphorylated protein or to a pair of reference spots (corners). The signal intensity is directly proportional to the amount of phosphorylation of the protein. B) Upon VEGF treatment of iPS-ECs, the phosphorylation state of determined proteins is different in Responders and Non-Responders. 


\section{Supplementary Figure 4}

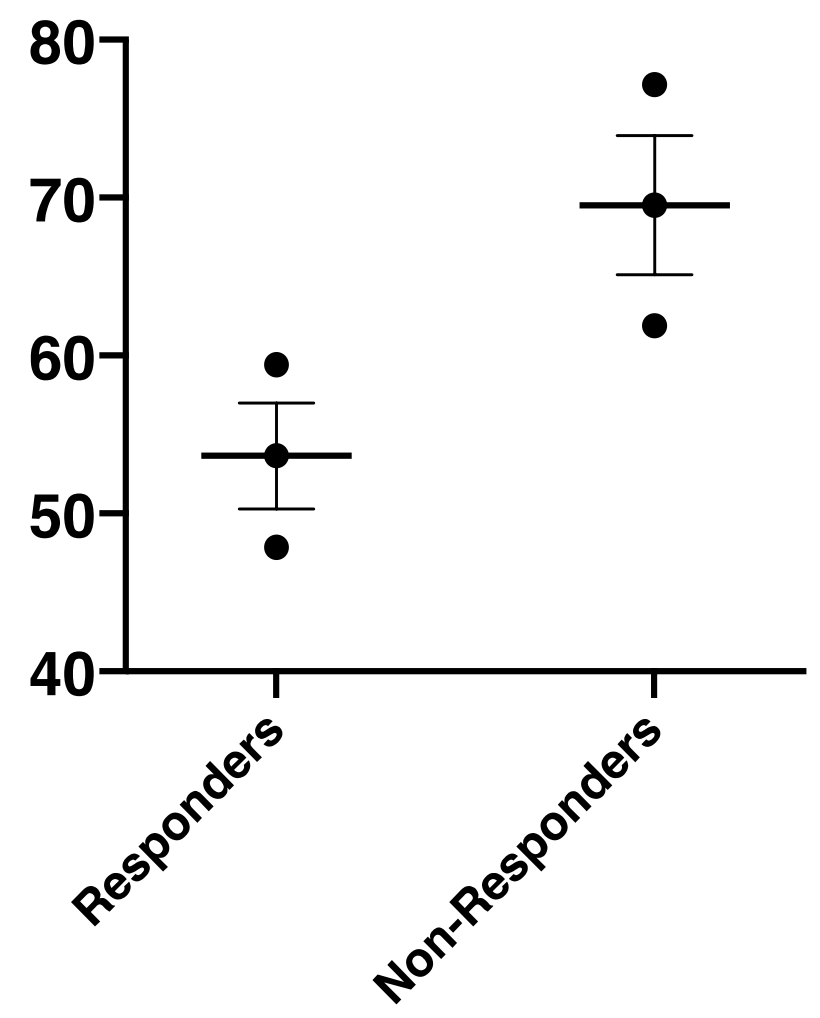

Supplementary Figure 4: FITC-Dextran relative \% diffusion levels in additional cell lines in Responders vs Non-Responders. (Data are means $\pm \operatorname{SEM}(n=3)$, $* * p<0.01)$. 
Supplementary Figure 5

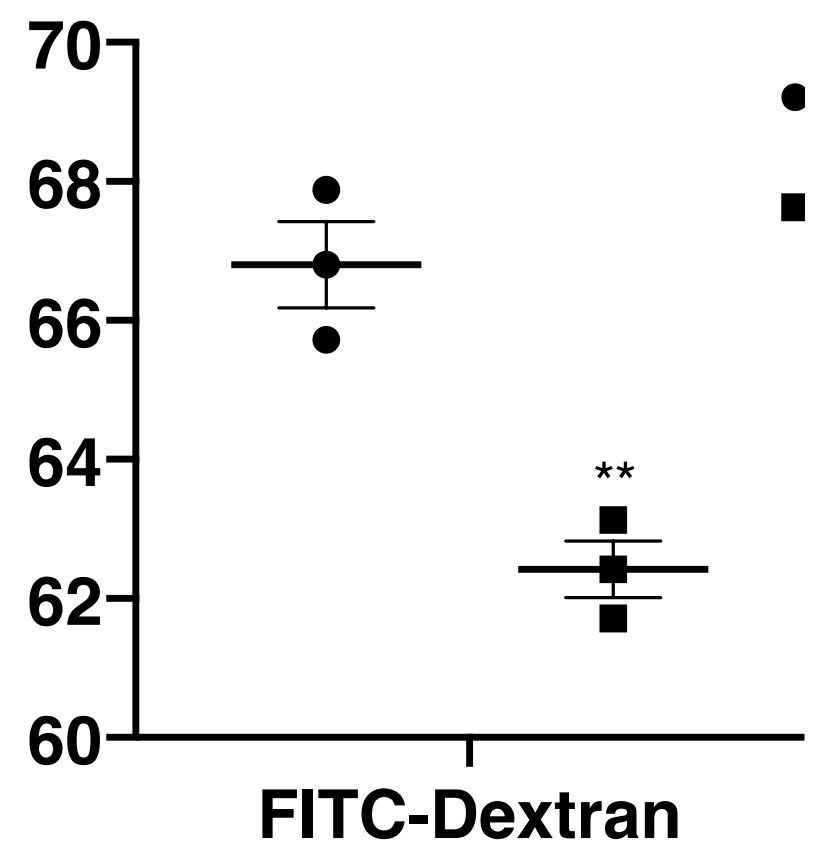

Supplementary Figure 5: FITC-Dextran relative \% diffusion levels after NPTX2 knockdown in additional cell lines of Non-Responders. (Data are means $\left.\pm \operatorname{SEM}(n=3),{ }^{* *} p<0.01\right)$. 
Figure 1

Vila Gonzalez et al.,

A
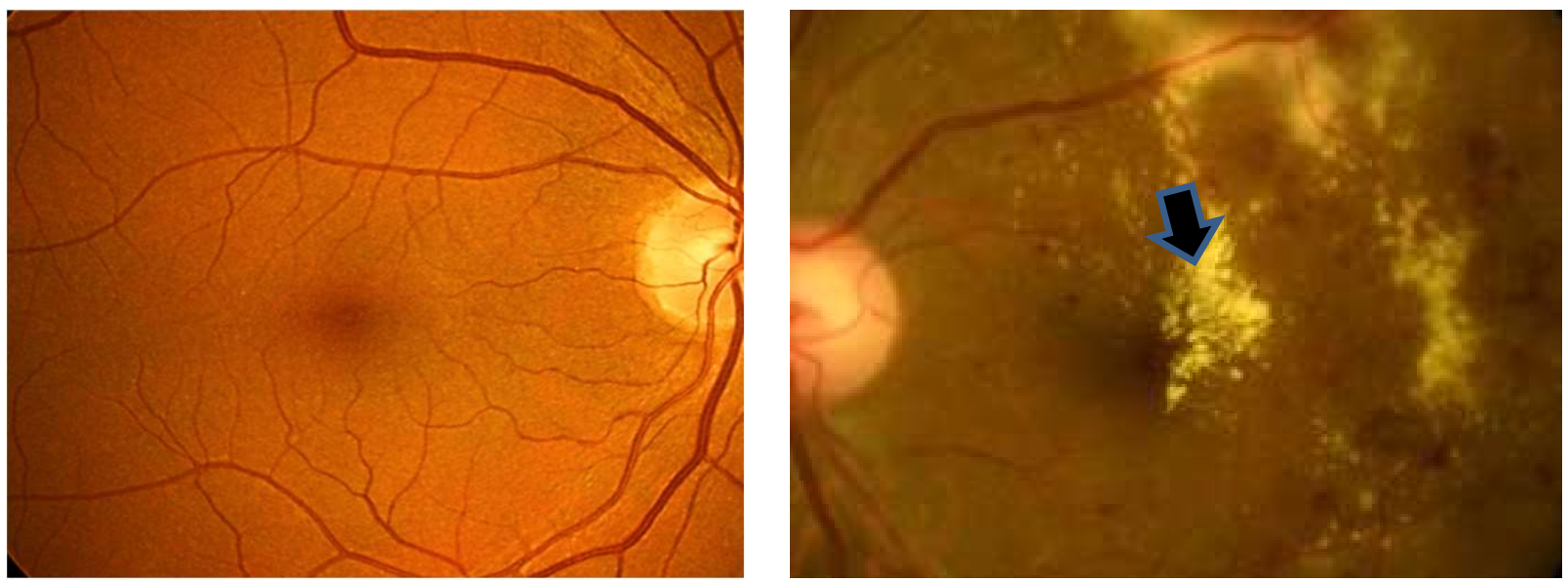

B

Baseline

Post- anti-VEGF treatment
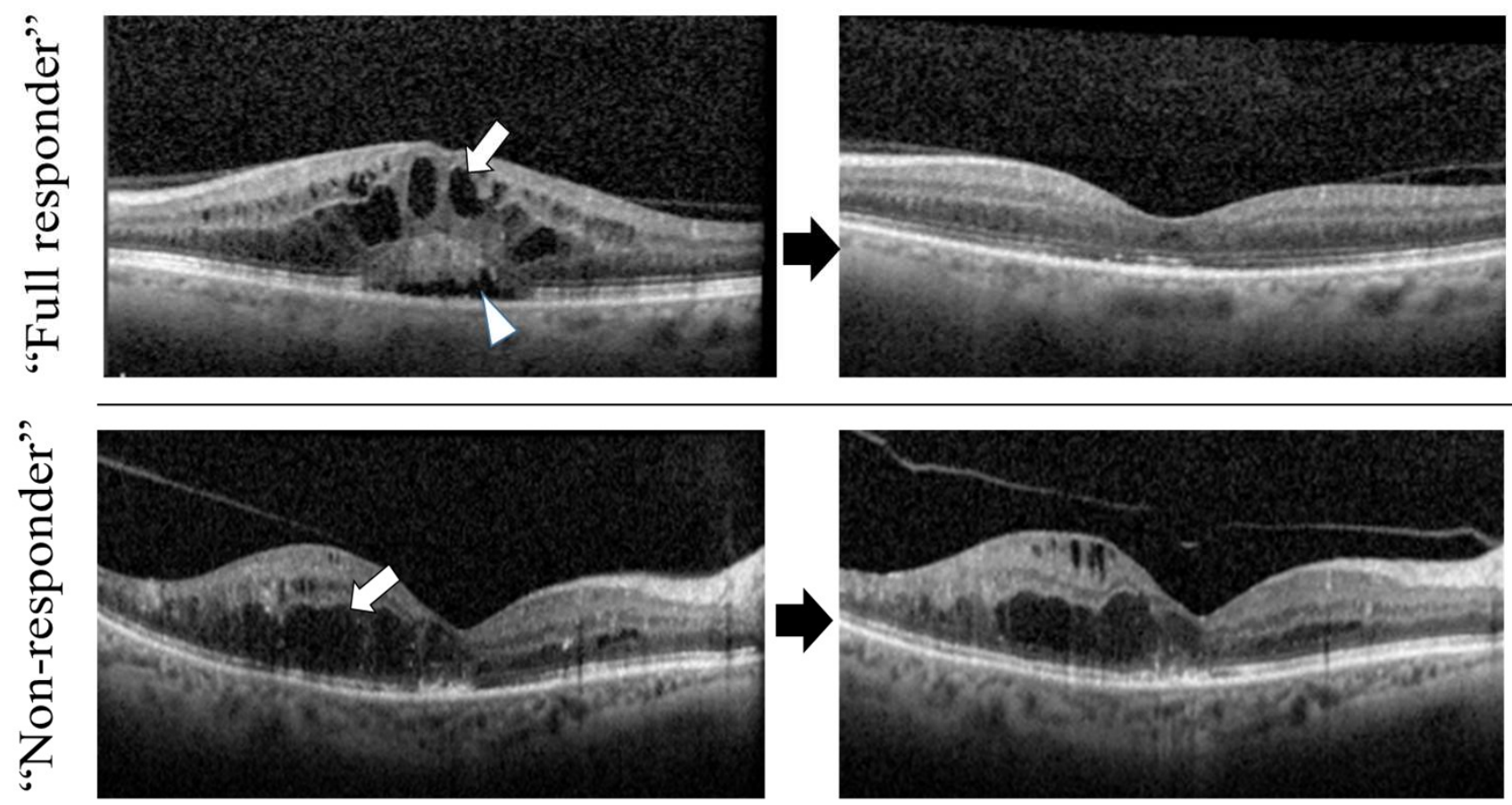
Figure 2

A

B
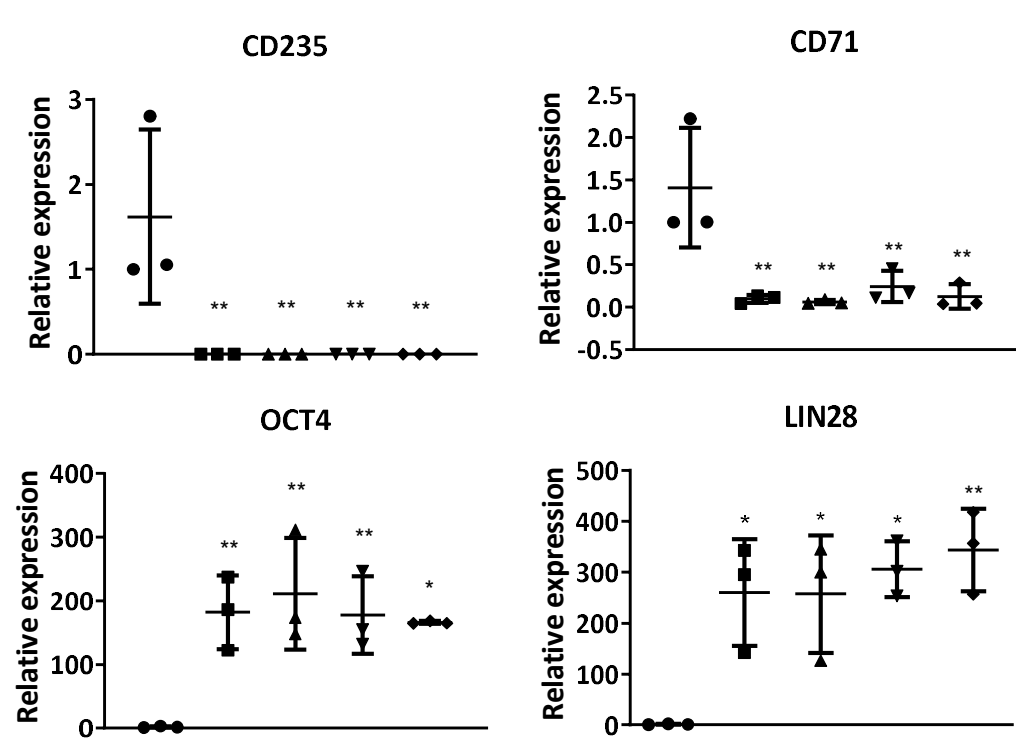

Vila Gonzalez et al.,

- $\mathrm{MNC}$

- Non-Diabetic

- Diabetic (mild DR)

v DME Responders

- DME Non-Responders
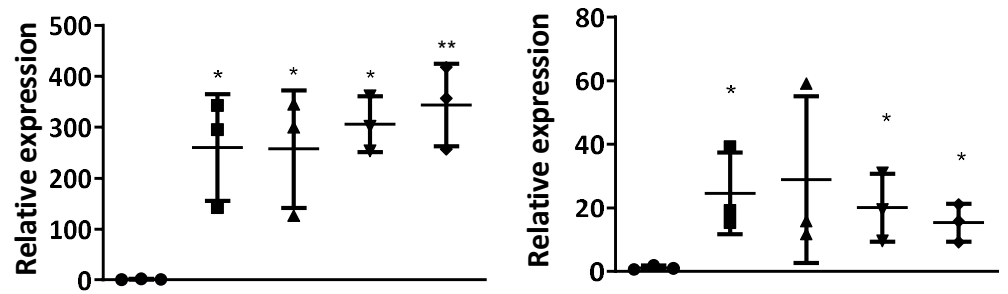

C

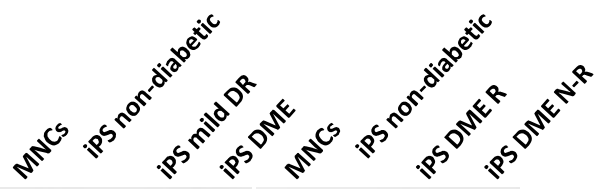

TRA-1-60

$45 \mathrm{kDa}$

Oct4

29 kDa

Lin 28

$42 \mathrm{kDa}$

$\boldsymbol{\beta}$ actin

D
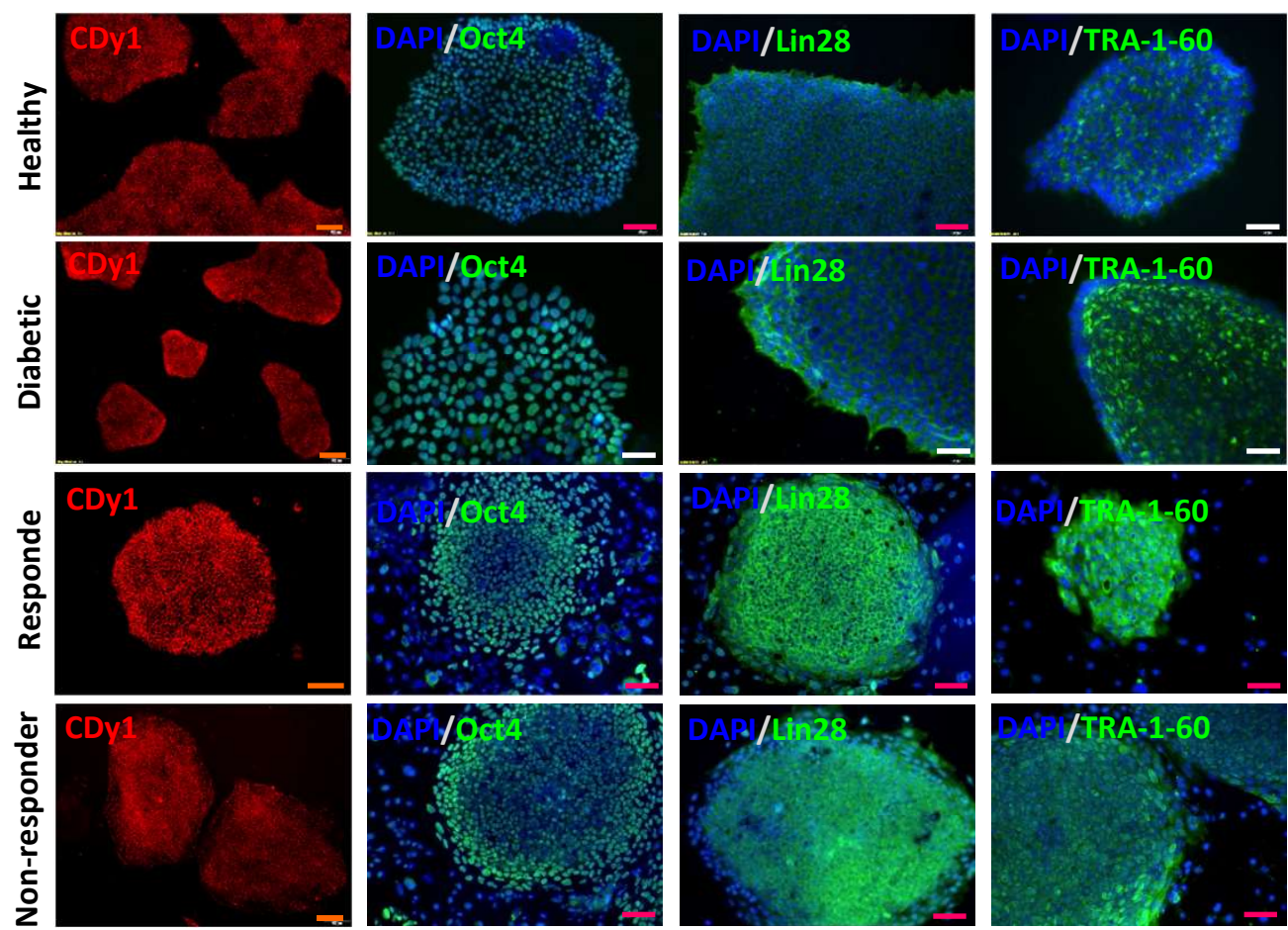
Figure 3

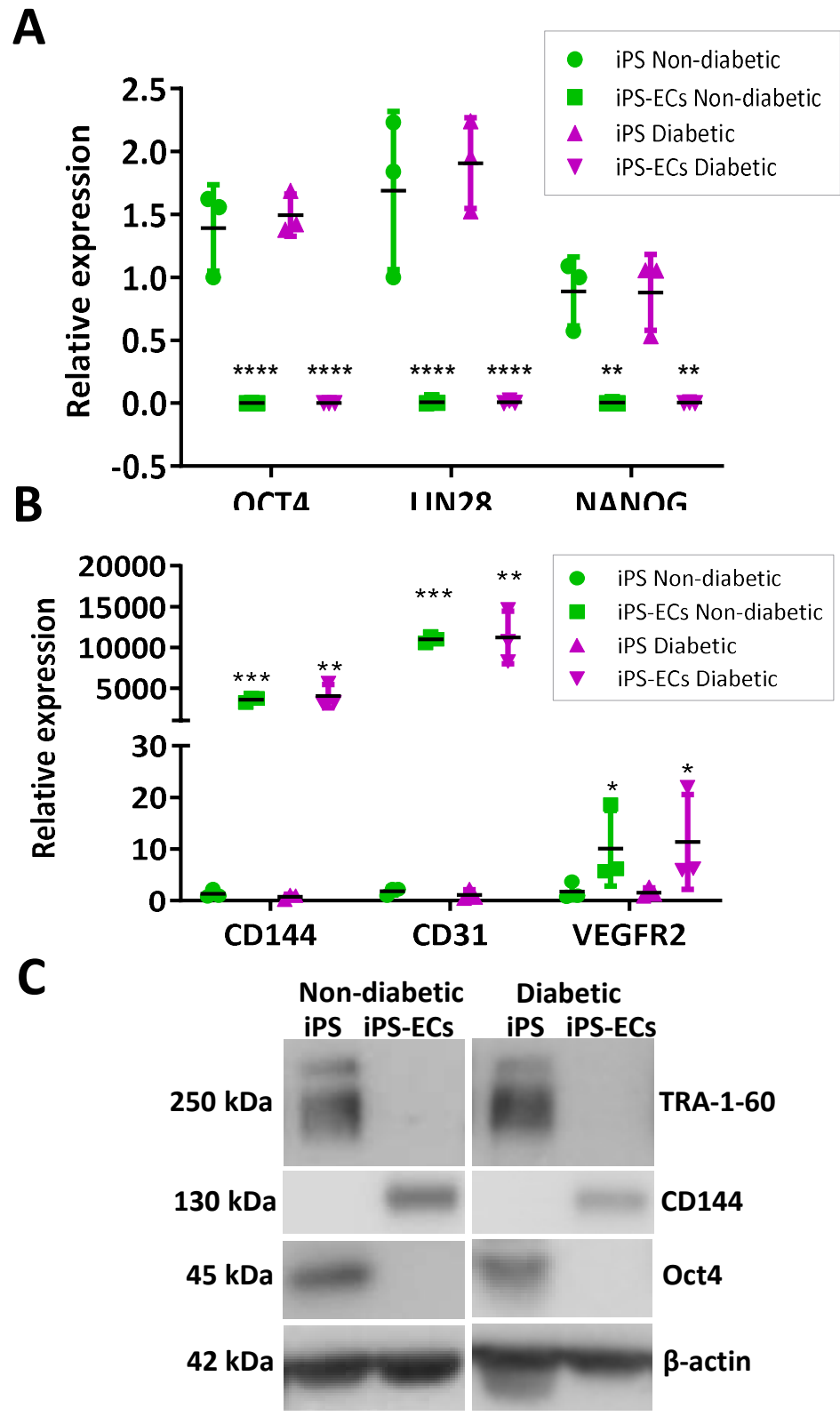

Vila Gonzalez et al.,

D Non-Diabetic
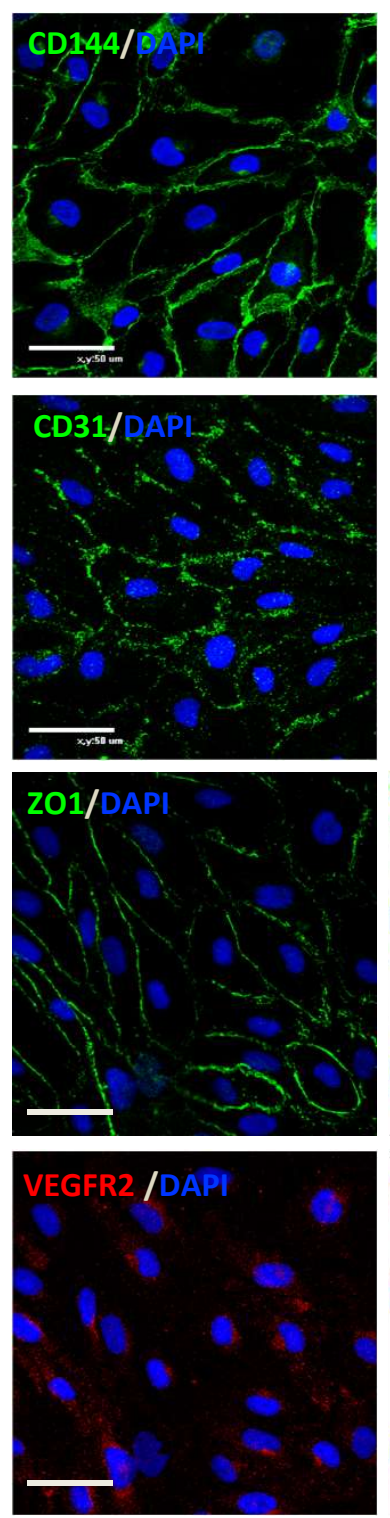

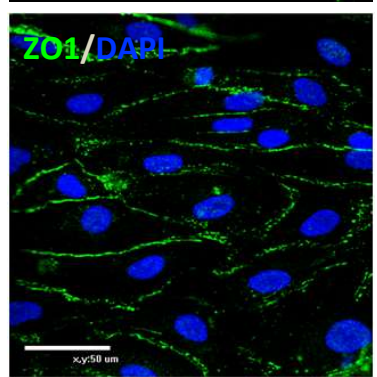

Diabetic
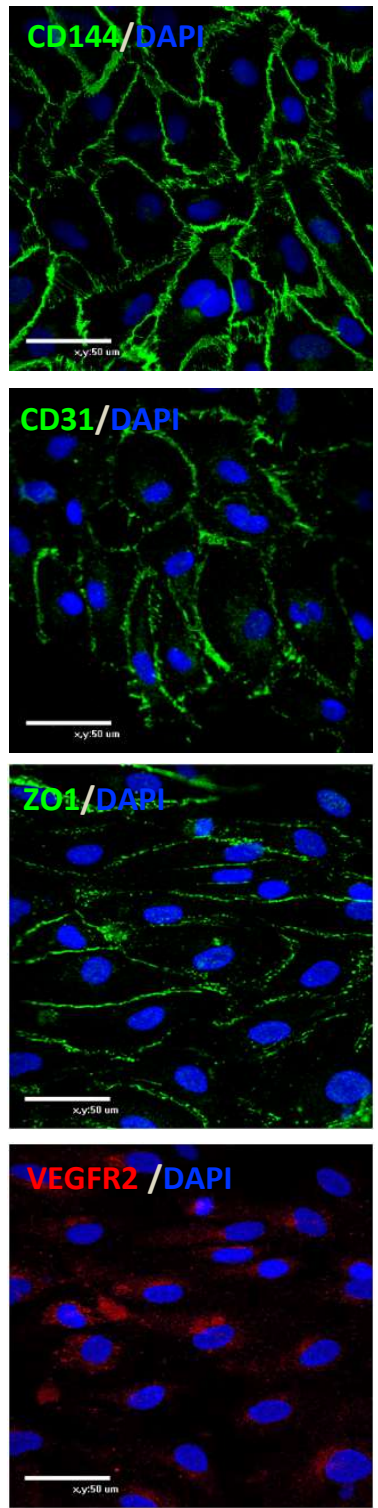
Figure 4

A

B
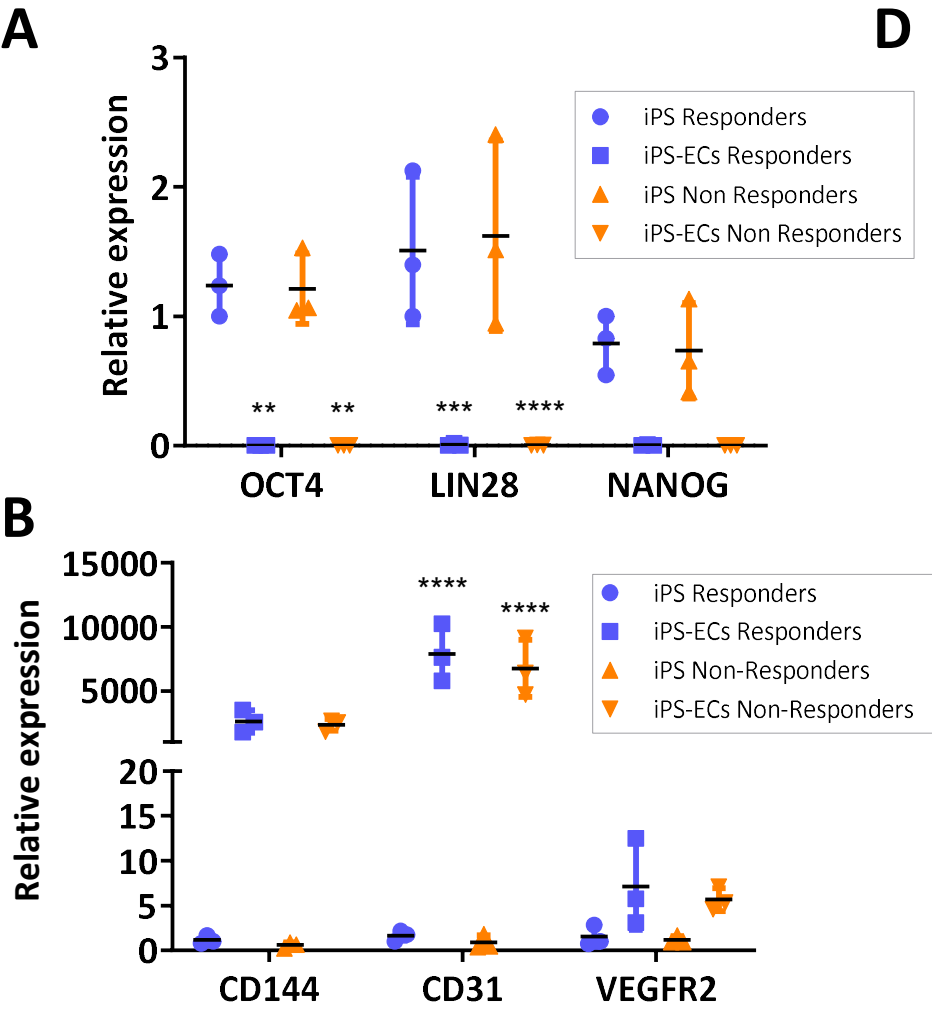

C
Vila Gonzalez et al.,
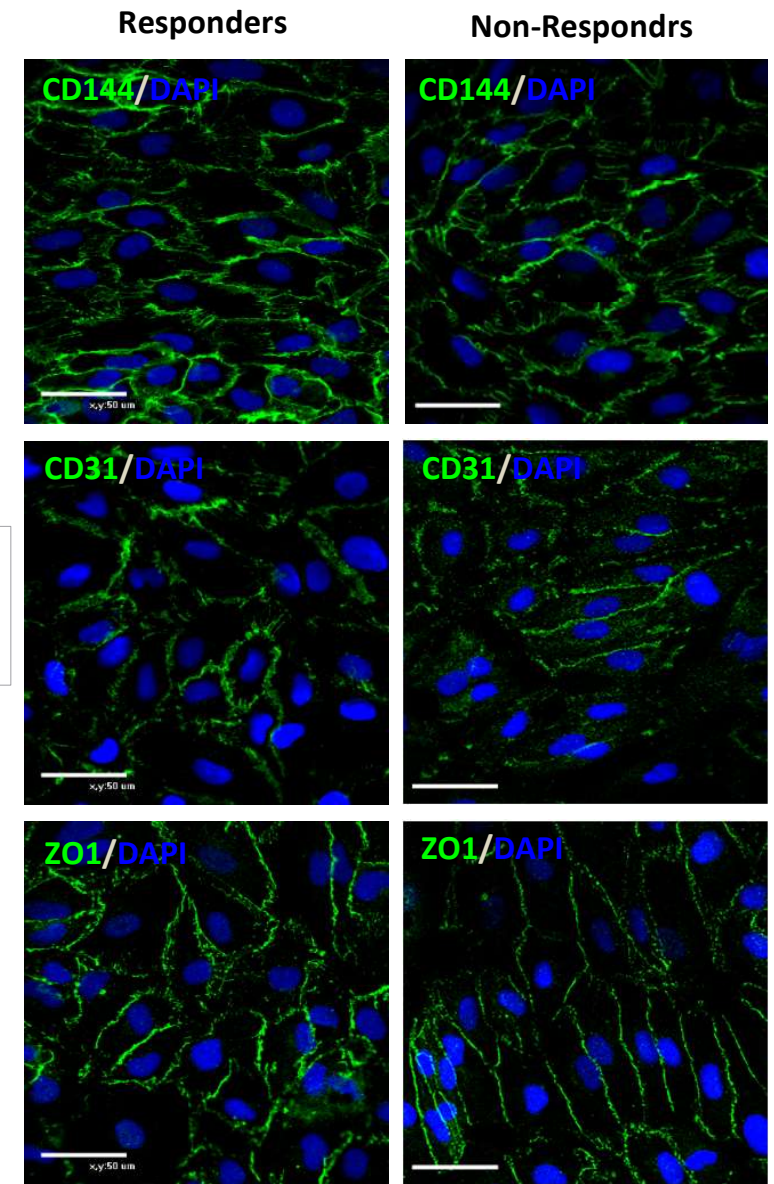

VEGFR2/

CD144

Oct4

$\beta$-actin

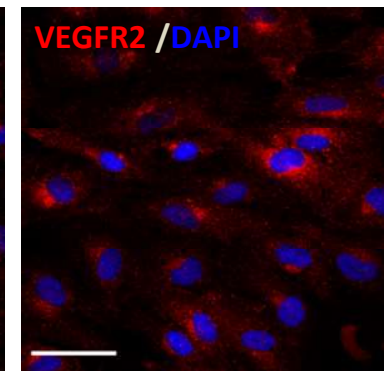


A

Before wound

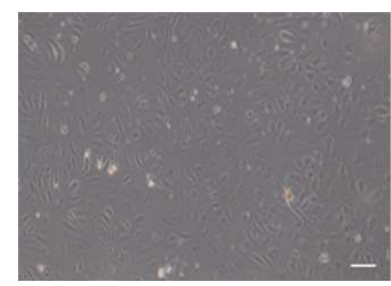

B
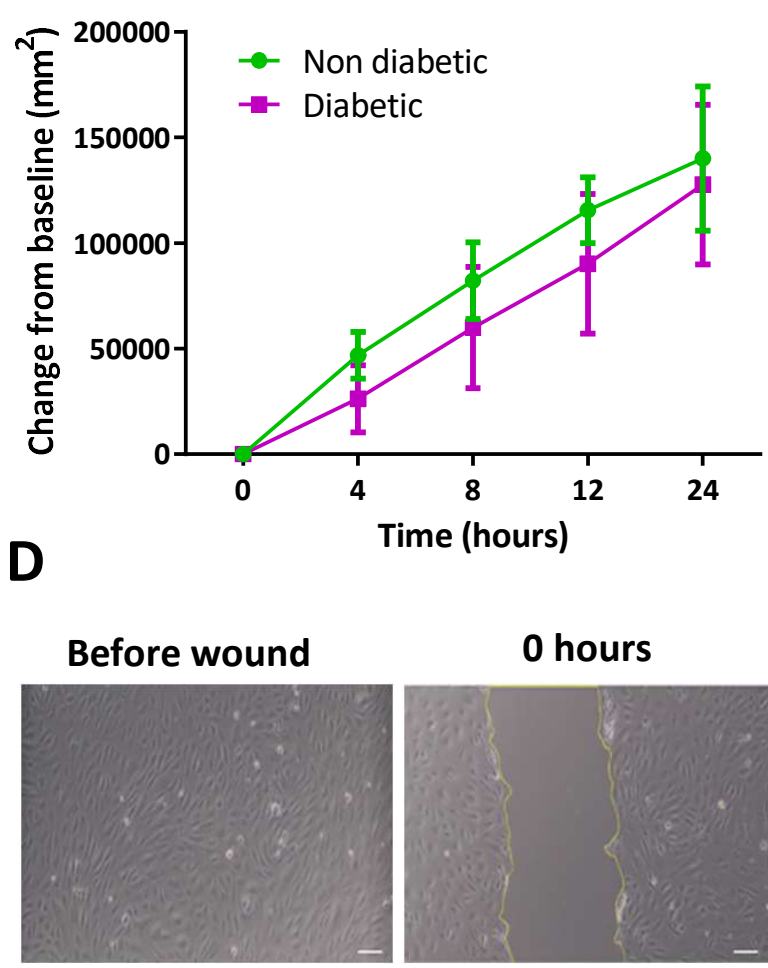

E

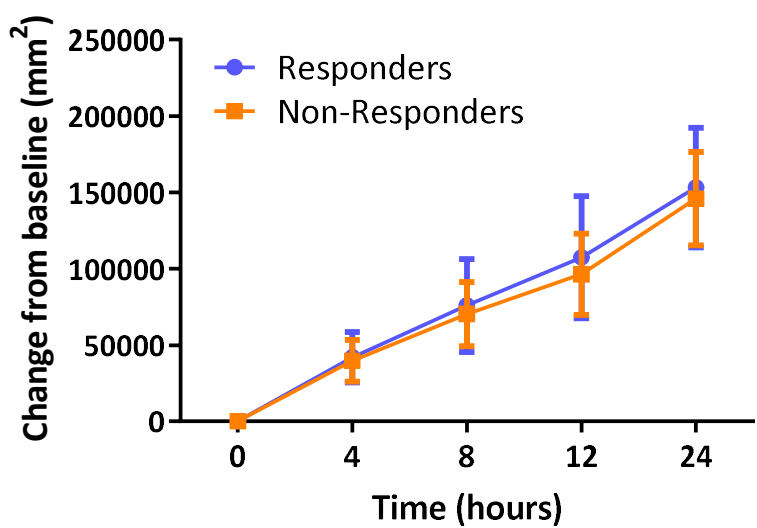

4 hours

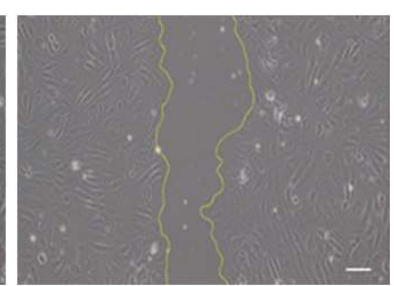

C

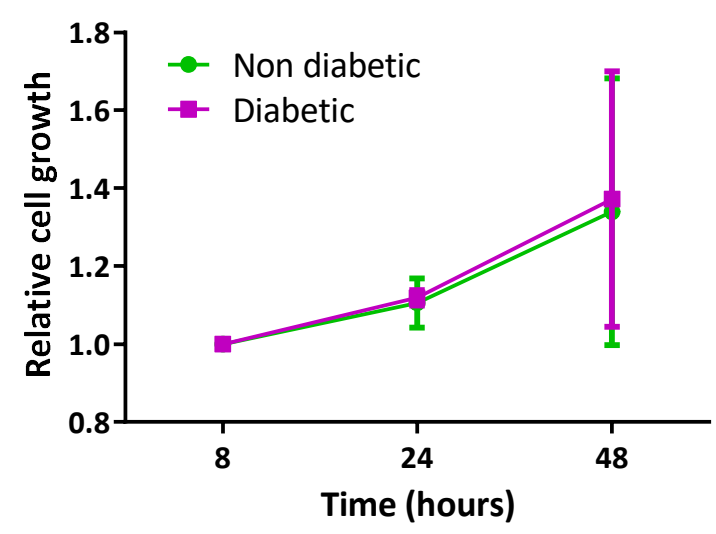

4 hours

8 hours
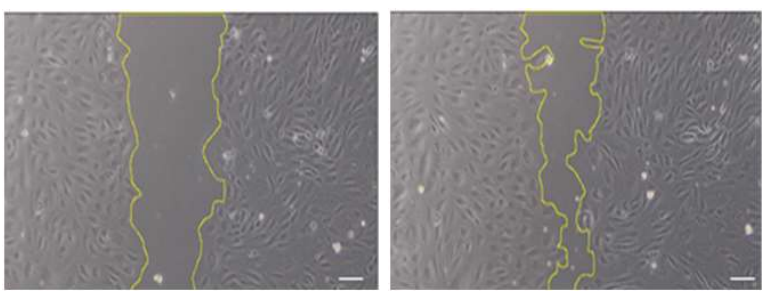

F

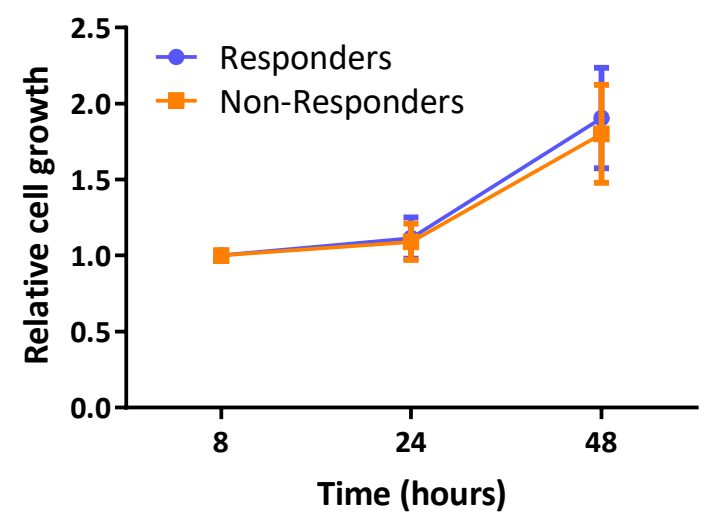


A

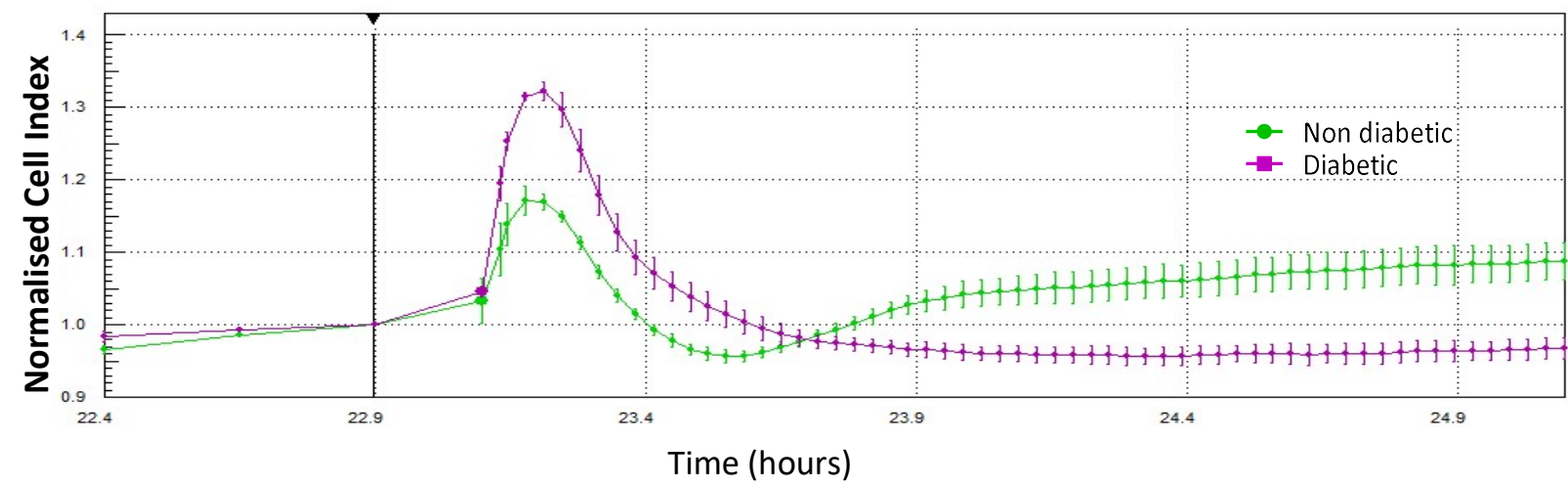

B Barrier integrity after VEGF treatment

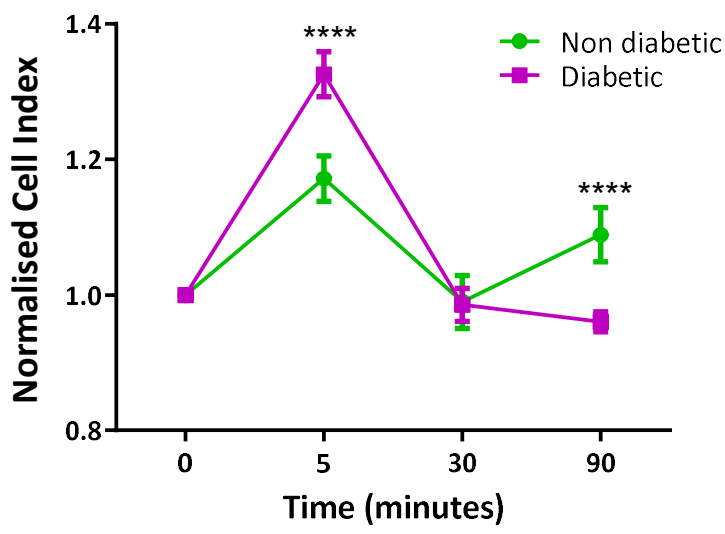

C

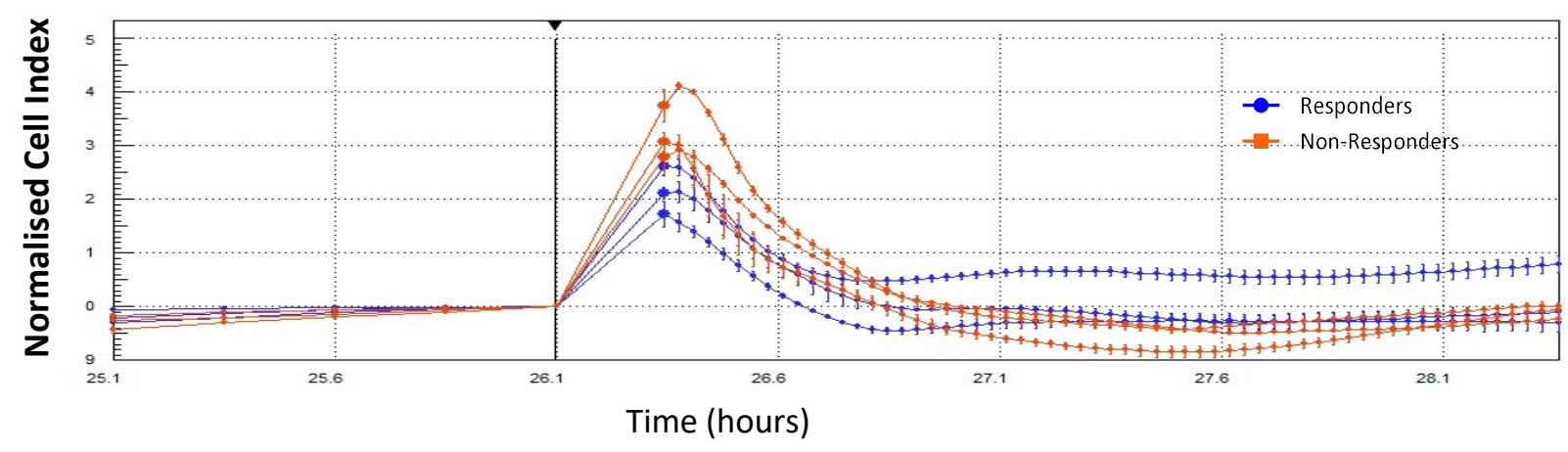

D

Barrier integrity after VEGF treatment

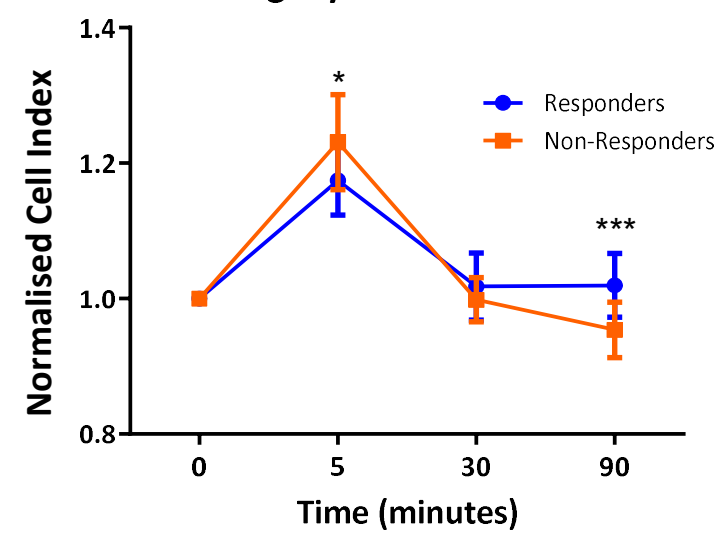


A

\begin{tabular}{llllll}
\multicolumn{2}{c}{ Responders } & & \multicolumn{3}{c}{ Non-Responders } \\
$0^{\prime} \quad 5^{\prime}$ & $20^{\prime}$ & & $0^{\prime}$ & $5^{\prime}$ & $20^{\prime}$
\end{tabular}

$250 \mathrm{kDa}$

P VEGFR2

$133 \mathrm{kDa}$

P eNOS

$42 \mathrm{kDa}$

$\beta$-actin

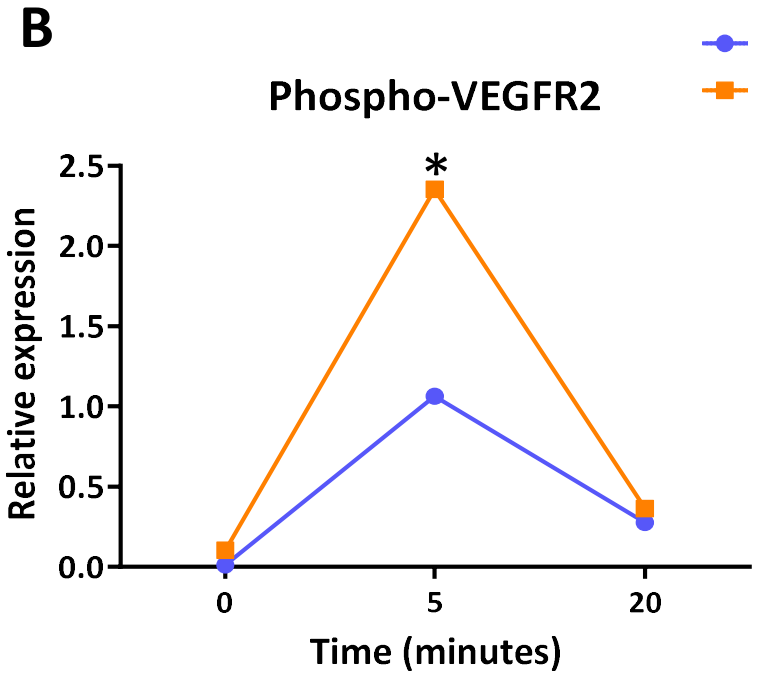

- Responders

- Non-Responders Phospho-eNOS

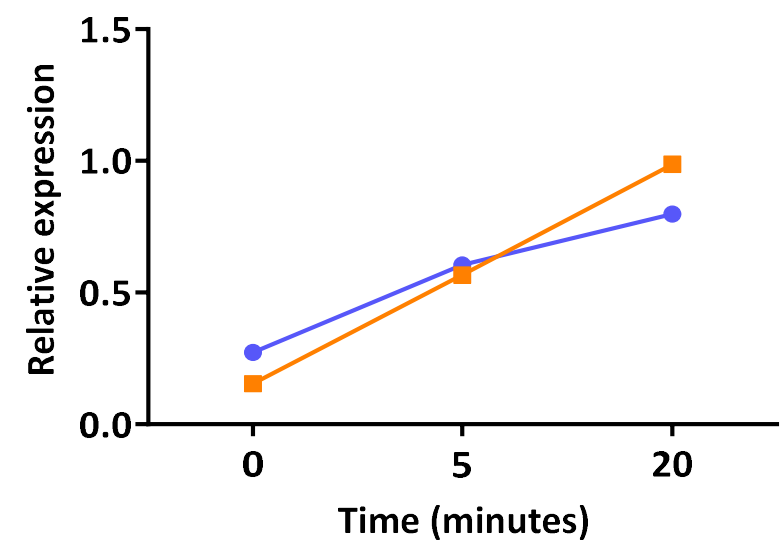


Figure 8

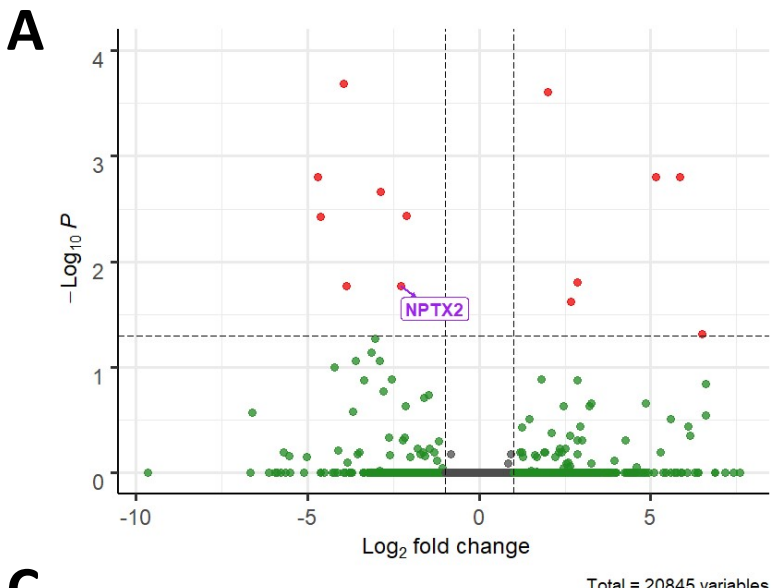

C

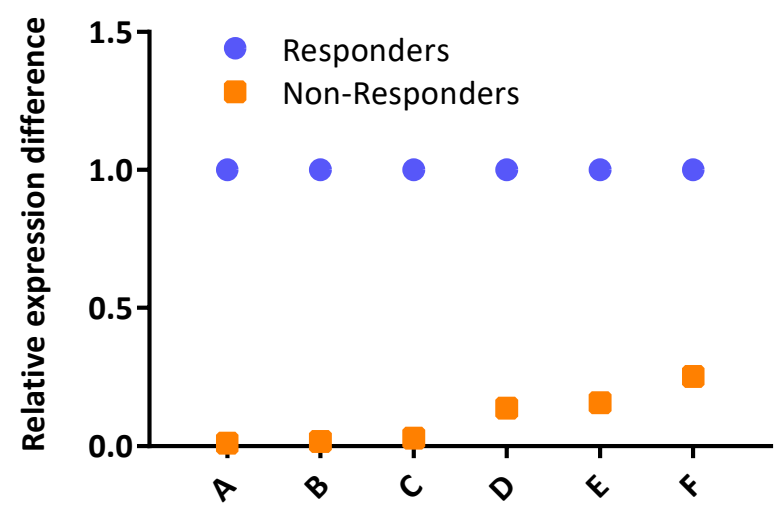

E

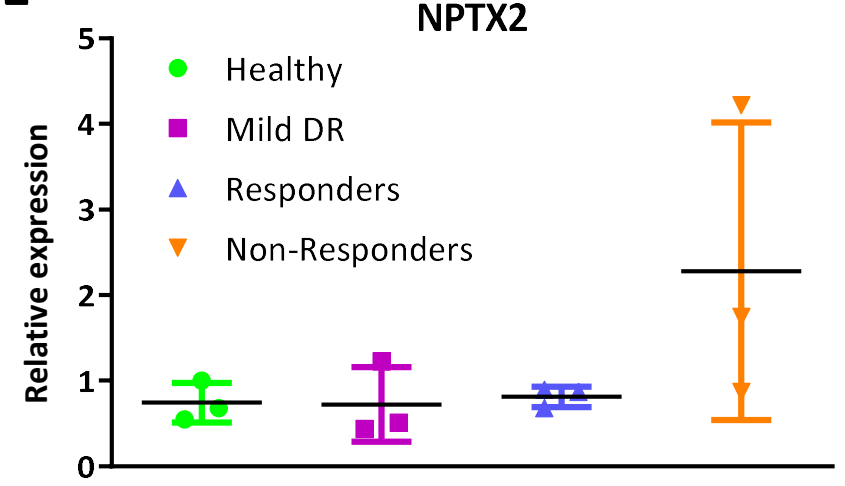

G

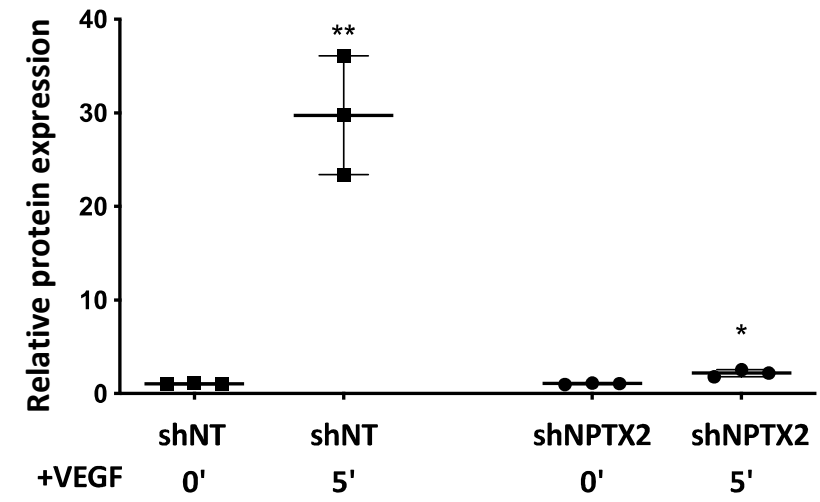

Vila Gonzalez et al.,

B

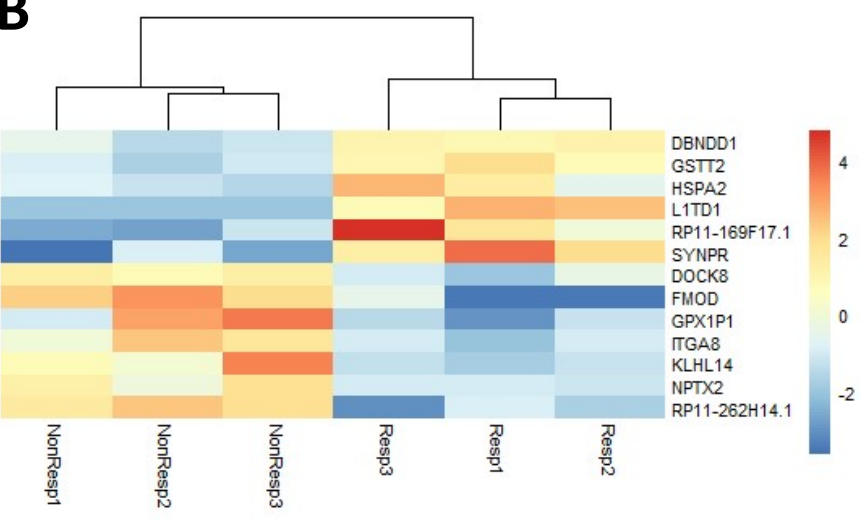

D

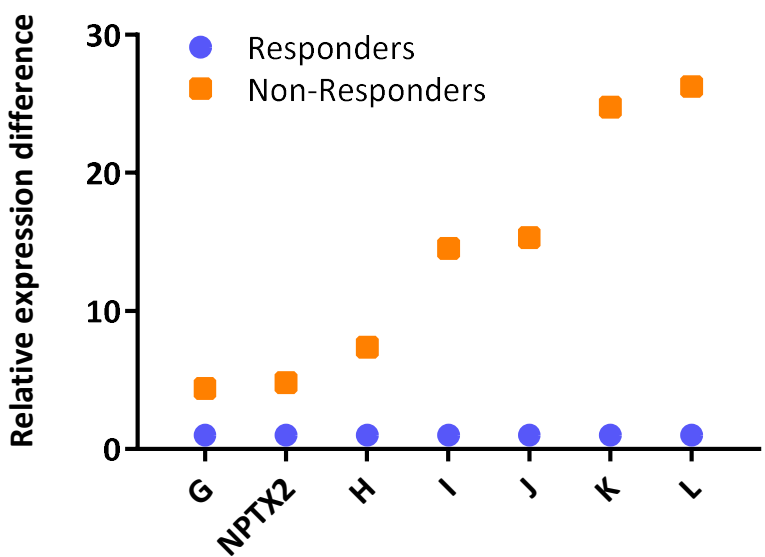

$\mathbf{F}$

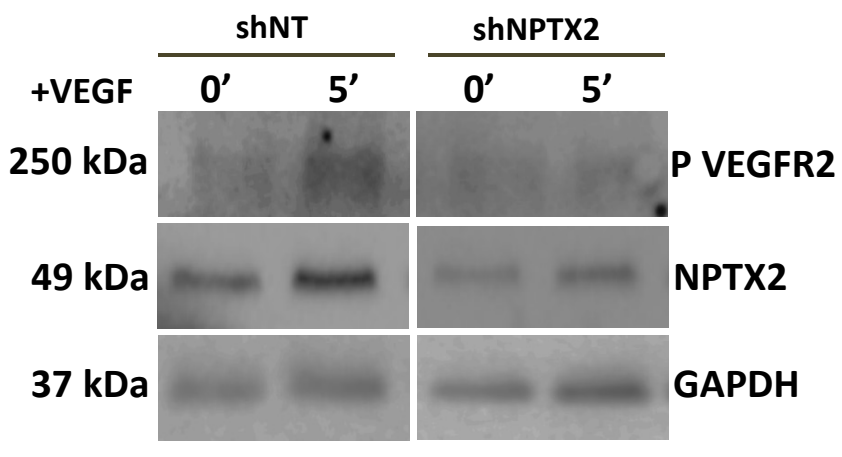

H

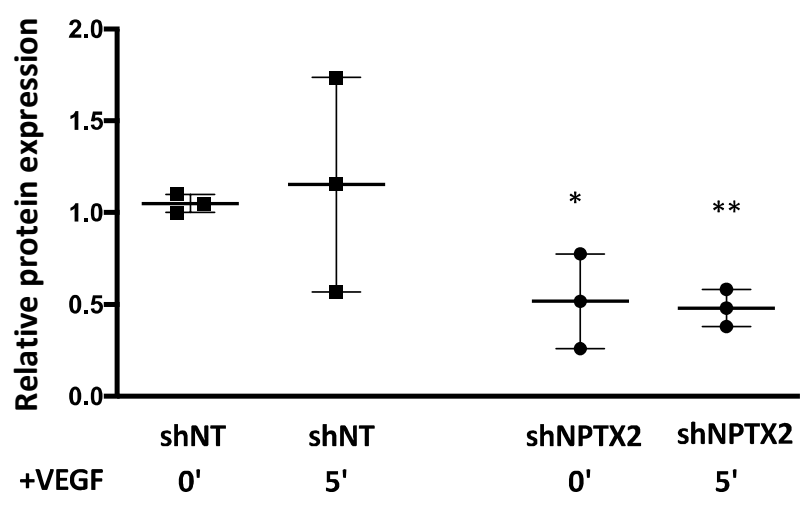

